MATHEMATICS OF COMPUTATION

Volume 66, Number 219, July 1997, Pages 1295-1321

S 0025-5718(97)00871-5

\title{
ZEROS OF DEDEKIND ZETA FUNCTIONS IN THE CRITICAL STRIP
}

\author{
EMMANUEL TOLLIS
}

\begin{abstract}
In this paper, we describe a computation which established the GRH to height 92 (resp. 40) for cubic number fields (resp. quartic number fields) with small discriminant. We use a method due to E. Friedman for computing values of Dedekind zeta functions, we take care of accumulated roundoff error to obtain results which are mathematically rigorous, and we generalize Turing's criterion to prove that there is no zero off the critical line. We finally give results concerning the GRH for cubic and quartic fields, tables of low zeros for number fields of degree 5 and 6 , and statistics about the smallest zero of a number field.
\end{abstract}

\section{INTRODUCTION AND NOTATIONS}

The Riemann zeta function and its generalization to number fields, the Dedekind zeta function, have been for well over a hundred years one of the central tools in number theory. It is recognized that the deepest single open problem in mathematics is the settling of the Riemann Hypothesis, and number theorists know that its generalization to number fields and algebraic varieties is almost equally important. Much energy has been devoted to the numerical investigation of the zeta function (see [4] for example). There has been some investigations of its closest cousins, the Dirichlet L-functions (see [20]). However, the case of a general number field has remained totally unexplored territory. We give here the first numerical evidence in favour of the Generalized Riemann Hypothesis for a number field where calculations cannot be reduced to the classical L-functions.

We explain how we have transformed a formula proved by Eduardo Friedman (in 1987) into an efficient algorithm for computing values of Dedekind zeta functions. The program (now included in the package Pari/GP) needs a few seconds to compute a single value of $\zeta_{K}$ for a number field of small degree. We also generalize Turing's criterion for Dedekind zeta functions to check the GRH.

In all the investigation, great care is taken to obtain results which are mathematically rigorous (estimate of error terms, of roundoff error...).

We finally give numerical results concerning the GRH. We have verified this hypothesis for 50 cubic number fields up to height 92 and for 30 quartic number fields up to height 40 . We give statistics about the gaps between zeros and about the height of the first zero of a number field.

Received by the editor January 20, 1996 and, in revised form, March 10, 1996.

1991 Mathematics Subject Classification. Primary 11R42.

Key words and phrases. Dedekind zeta function, Generalized Riemann Hypothesis.

(C)1997 American Mathematical Society 
In all, the computations represent between two and three months of running time on a Sparc-10 machine (see 3.4).

We use the following notations. We let $K$ be a number field of degree $N$ and signature $\left(r_{1}, r_{2}\right), \mathcal{O}_{K}$ its ring of integers, and $D_{K}$ its discriminant. We also use the regulator $R_{K}$, the class number $h_{K}$, and the number $\omega_{K}$ of roots of unity. The Dedekind zeta function of this field is of course defined by

$$
\zeta_{K}(s)=\sum_{\mathfrak{a} \subset \mathcal{O}_{K}}(\mathcal{N} \mathfrak{a})^{-s}=\sum_{n \geq 1} a_{n} n^{-s} \quad \text { for } \operatorname{Re} s>1
$$

where $a_{n}$ is the number of ideals with norm equal to $n$. This function is extended by analytic continuation to a meromorphic function on the whole complex plane with a unique simple pole at $s=1$. Moreover, $\zeta_{K}$ verifies a functional equation:

$$
\Lambda_{K}(s)=\Lambda_{K}(1-s)
$$

where

$$
\Lambda_{K}(s)=\Gamma\left(\frac{s}{2}\right)^{r_{1}} \Gamma(s)^{r_{2}}\left(\frac{\sqrt{\left|D_{K}\right|}}{\pi^{\frac{N}{2}} 2^{r_{2}}}\right)^{s} \zeta_{K}(s)
$$

and $\Lambda_{K}$ is meromorphic on the complex plane with two poles at $s=1$ and at $s=0$.

\section{The METhod FOR COMPUTING VAlues}

In [7], Eduardo Friedman explains how we can simplify the multiple integral appearing in Hecke's formula by turning it into a single integral. The main result that we will need is the following (see Proposition 2.3 and Theorem 1 in [7]).

\section{Theorem 1.1.}

$$
\Lambda_{K}(s)=\frac{2^{r_{1}} h_{K} R_{K}}{\omega_{K}(s-1) s}+\sum_{n \geq 1} a_{n}\left[f\left(\frac{C_{K}}{n}, s\right)+f\left(\frac{C_{K}}{n}, 1-s\right)\right]
$$

with the notations of Section 0 and

$$
C_{K}=\frac{\sqrt{\left|D_{K}\right|}}{\pi^{\frac{N}{2}} 2^{r_{2}}}
$$

$$
f(x, s)=\frac{1}{2 i \pi} \int_{\delta-i \infty}^{\delta+i \infty} x^{z} \Gamma\left(\frac{z}{2}\right)^{r_{1}} \Gamma(z)^{r_{2}} \frac{d z}{z-s}, \quad \delta>\max (\operatorname{Re} s, 0) .
$$

Apart from the problem of computing the regulator and the class number of a number field (which is made at the beginning of the program since it is independent of $s$ ), we must also compute the function $f$ at a great number of points. For this, we shift to the left the line of integration, and we compute the residues of the integrand at the poles that we encounter (at $z=s$ and at negative integers). We thus obtain the following result. 


\section{Proposition 1.1.}

$f(x, s)=\sum_{j=1}^{r_{1}+r_{2}} \sum_{i \geq 0} A_{i, j}(s) x^{-i} \frac{(\ln x)^{j-1}}{(j-1) !}+x^{s} \Gamma\left(\frac{s}{2}\right)^{r_{1}} \Gamma(s)^{r_{2}}$

if $s$ is not a negative integer,

$$
f(x, s)=\sum_{j=1}^{r_{1}+r_{2}} \sum_{\substack{i \geq 0 \\ i \neq-s}} A_{i, j}(s) x^{-i} \frac{(\ln x)^{j-1}}{(j-1) !}+\sum_{j=1}^{r_{1}+r_{2}+1} A_{-s, j}(s) x^{s} \frac{(\ln x)^{j-1}}{(j-1) !}
$$

otherwise,

where the coefficients $A_{i, j}(s)$ are defined by

$$
\frac{\Gamma\left(\frac{z}{2}\right)^{r_{1}} \Gamma(z)^{r_{2}}}{z-s}=\sum_{j=1}^{r_{1}+r_{2}+1} \frac{A_{i, j}(s)}{(z+i)^{j}}+\text { a function analytic at } z=-i .
$$

Proof. The proof of this result is quite easy and is done in [7] for the first case. In the second case, $s$ is a negative integer and so, the function $\frac{\Gamma(z / 2)^{r_{1}} \Gamma(z)^{r_{2}}}{z-s}$ has a pole of order $r_{1}+r_{2}+1$ (instead of $r_{1}+r_{2}$ ) at $z=s$. In this case, the computation of the coefficients $A_{-s, j}(s)$ is also slightly different (see the next section).

\section{Computation of the Coefficients}

2.1. The number $a_{n}$ of ideals of norm $n$. We compute the regulator, the class number and the numbers $a_{n}$ at the beginning of the program since it only depends on the number field. The best method that we have found for computing the $a_{n}$ is to use the Euler product as follows. If $\mathcal{P}$ is the set of primes of $\mathbb{Z}$, we have

$$
\sum_{n \geq 1} a_{n} n^{-s}=\prod_{p \in \mathcal{P}} \prod_{\mathfrak{p} / p}\left(1-\frac{1}{(\mathcal{N} \mathfrak{p})^{s}}\right)^{-1} \quad \text { if } \operatorname{Re} s>1 .
$$

Suppose we want to compute the coefficients $a_{n}$ for $n=1$ to $n=N_{0}$ for a given $N_{0}$, and suppose also that we have numbered all the prime ideals $\mathfrak{p}_{i}(i=1, \ldots, m)$ such that $\mathcal{N} \mathfrak{p}_{i} \leq N_{0}$. Let $f_{i}=f_{\mathfrak{p}_{i}}$ be the residual degree of $\mathfrak{p}_{i}$ and $p_{i}$ the prime number below $\mathfrak{p}_{i}$. We compute the $a_{n}$ recursively, introducing the coefficients $a_{n, h}$ (for $h=0, \ldots, m$ ) by

$$
\left\{\begin{array}{l}
a_{1,0}=1 \quad \text { and } \quad a_{n, 0}=0 \quad \text { for } n \geq 2 \\
\sum_{n=1}^{\infty} a_{n, h} n^{-s}=\prod_{i=1}^{h} \sum_{k \geq 0} \frac{1}{p_{i}^{k f_{i} s}} \quad \text { if } h \geq 1 .
\end{array}\right.
$$

With a little computation, we have

$$
a_{n, h}=\sum_{k=0}^{v} a_{\frac{n}{P^{k}}, h-1}
$$

where $P=p_{h}^{f_{h}}$, and $v$ is the largest integer such that $P^{v}$ divides $n$. We finally obtain the coefficients $a_{n}$ since $a_{n, m}=a_{n}$ for $n \leq N_{0}$. 
2.2. The coefficients $A_{i, j}(s)$. Computing these coefficients is the main problem for us (see the formulas in Proposition 1.1). The best solution that we have found is to introduce coefficients $A_{i, j}$ which are independent of $s$ and depend only on the number field. They can be computed at the beginning of the program. For each value of $s$, we will then deduce recursively the $A_{i, j}(s)$ from the $A_{i, j}$. We define

$$
\Gamma\left(\frac{z}{2}\right)^{r_{1}} \Gamma(z)^{r_{2}}=\sum_{j=0}^{r_{1}+r_{2}} \frac{A_{i, j}}{(z+i)^{j}}+\text { a function analytic at } z=-i
$$

and a little computation gives the following result on the $A_{i, j}$.

Proposition 2.1. Let $H_{q, k}=\sum_{j=1}^{q} \frac{1}{j^{k}}$, let $\delta_{1, k}$ be the Dirac symbol, and by abuse of notation, let $\zeta(1)=\gamma$ (Euler's constant).

1) For $i=2 q$ even we have for $0<|z+2 q|<1$,

$$
\begin{aligned}
& \Gamma\left(\frac{z}{2}\right)^{r_{1}} \Gamma(z)^{r_{2}}=\frac{(-1)^{q r_{1}} 2^{r_{1}}}{q^{!_{1}}(2 q) !^{r_{2}}(z+2 q)^{r_{1}+r_{2}}} \exp [g(z)], \quad \text { where } \\
& g(z)=\sum_{k \geq 1} \frac{(z+2 q)^{k}}{k}\left[(-1)^{k} \zeta(k)\left(\frac{r_{1}}{2^{k}}+r_{2}\right)+\frac{r_{1}}{2^{k}} H_{q, k}+r_{2} H_{2 q, k}\right] .
\end{aligned}
$$

2) For $i=2 q+1$ odd we have for $0<|z+2 q+1|<1$,

$$
\begin{aligned}
& \Gamma\left(\frac{z}{2}\right)^{r_{1}} \Gamma(z)^{r_{2}}=\frac{(-1)^{(q+1) r_{1}+r_{2}} \pi^{\frac{r_{1}}{2}} 2^{(2 q+1) r_{1}} q^{! r_{1}}}{(2 q+1) !^{r_{1}+r_{2}}(z+2 q+1)^{r_{2}}} \exp [g(z)], \text { where } \\
& g(z)=\sum_{k \geq 1} \frac{(z+2 q+1)^{k}}{k}\left[(-1)^{k} \zeta(k)\left(r_{1}\left(1-\frac{1}{2^{k}}\right)+r_{2}\right)\right. \\
& \left.\quad+\left(r_{1}+r_{2}\right) H_{2 q+1, k}-\frac{r_{1}}{2^{k}} H_{q, k}-r_{1} \delta_{1, k} \ln 2\right] .
\end{aligned}
$$

Proof. For the first part, we let $X=z+2 q$, then $\Gamma(X-2 q)=\frac{\Gamma(X+1)}{(X-2 q)(X-2 q+1) \ldots X}$ and so

$$
\begin{aligned}
\ln \left[\Gamma\left(\frac{z}{2}\right)^{r_{1}} \Gamma(z)^{r_{2}}\right]= & r_{1} \ln \Gamma\left(\frac{X}{2}+1\right)+r_{2} \ln \Gamma(X+1) \\
& -r_{1} \ln \left[\left(\frac{X}{2}-q\right) \ldots \frac{X}{2}\right]-r_{2} \ln [(X-2 q) \ldots X] .
\end{aligned}
$$

We thus have

$$
\begin{aligned}
\ln \left[\Gamma\left(\frac{z}{2}\right)^{r_{1}} \Gamma(z)^{r_{2}}\right]= & \sum_{k \geq 1} \frac{X^{k}}{k}(-1)^{k} \zeta(k)\left(\frac{r_{1}}{2^{k}}+r_{2}\right)-r_{1} \ln \left[(-1)^{q} q ! \frac{X}{2}\right] \\
& -r_{1} \sum_{j=1}^{q} \ln \left(1-\frac{X}{2 j}\right)-r_{2} \ln ((2 q) ! X)-r_{2} \sum_{j=1}^{2 q} \ln \left(1-\frac{X}{j}\right) \\
= & \sum_{k \geq 1} \frac{X^{k}}{k}\left[(-1)^{k} \zeta(k)\left(\frac{r_{1}}{2^{k}}+r_{2}\right)+\frac{r_{1}}{2^{k}} H_{q, k}+r_{2} H_{2 q, k}\right] \\
& -r_{1} \ln \left[(-1)^{q} q ! \frac{X}{2}\right]-r_{2} \ln [(2 q) ! X]
\end{aligned}
$$


(see [1] for the expansion formula of $\ln \Gamma(1+z)$ ). To prove (12), we let $X=z+2 q+1$ and we have

$$
\Gamma\left(\frac{X-2 q-1}{2}\right)=\frac{\Gamma(X+1) \sqrt{\pi}\left(\frac{X}{2}-q-1\right) \ldots\left(\frac{X}{2}-1\right)}{\Gamma\left(\frac{X}{2}+1\right) 2^{X-2 q-2}(X-2 q-2) \ldots(X-1)} .
$$

We compute the logarithm of this expression, we find

$$
\begin{aligned}
\ln \Gamma\left(\frac{X-2 q-1}{2}\right)= & \sum_{k \geq 1} \frac{X^{k}}{k}\left[(-1)^{k} \zeta(k)\left(1-\frac{1}{2^{k}}\right)+H_{2 q+1, k}-\frac{H_{q, k}}{2^{k}}-\delta_{k, 1} \ln 2\right] \\
& +\ln q !-\ln (2 q+1) !+\ln \sqrt{\pi}+(2 q+1) \ln 2+\ln (-1)^{q+1}
\end{aligned}
$$

and after a computation we obtain formula (12).

In practice, we compute the coefficients of the expansion of the function $g$ to $r_{1}+r_{2}+1$ terms if $i$ is odd and to $r_{2}+1$ terms if $i$ is even. After exponentiating the series we obtain the coefficients $A_{i, j}$. It is clear that many terms in the expressions (11) and (12) can be recursively computed. For a given value of $s$, we can now deduce the coefficients $A_{i, j}(s)$ from the preceding results. Formulas (7) and (10) give immediately the following relations between $A_{i, j}$ and $A_{i, j}(s)$.

$$
\begin{aligned}
& \text { if } i \neq-s \quad A_{i, j}(s)=\frac{A_{i, j+1}(s)-A_{i, j}}{s+i} \text { with } \\
& \left\{\begin{array}{l}
A_{i, r_{1}+r_{2}+1}(s)=0 \quad \text { if } i \text { is even, } \\
A_{i, r_{2}+1}(s)=0 \quad \text { if } i \text { is odd, }
\end{array}\right. \\
& \text { if } i=-s \quad A_{-s, j}(s)=A_{-s, j-1} .
\end{aligned}
$$

2.3. Another form of the method. Our first goal in this paper is to check numerically the Generalized Riemann Hypothesis. For that, we need to locate the zeros of $\zeta_{K}$, and hence we must compute thousands of values with our program. As a consequence, it must be as fast as possible. The best solution that we have found for doing this is to separate the computations in two parts. First, at the beginning of the program, we compute all the data which are independent of the value of $s$. These are data linked to the number field (discriminant, regulator, class number,...) and the coefficients $A_{i, j}$. Hence, when the value of $s$ is known, we have a minimal number of operations to perform.

In a first version of the program, we simply used all the preceding results without any change. But, with little difficulty, we can improve the method by a simple inversion of summations. Nevertheless, this slight modification implies the need for rigorous verifications about the size of the coefficients (see Section 5). Here are the final formulas.

Proposition 2.2. Let $\epsilon$ be the error made truncating the series (3) and (5) to $N_{0}$ and $i_{0}$ terms (we will see in 3.2 and 3.3 that $N_{0}$ and $i_{0}$ depend only on the number 
field and on the absolute precision required). If $s$ is not an integer

$$
\begin{aligned}
& \Lambda_{K}(s)=\frac{2^{r_{1}} h_{K} R_{K}}{\omega_{K}(s-1) s}+C_{K}^{s} \Gamma\left(\frac{s}{2}\right)^{r_{1}} \Gamma(s)^{r_{2}} \sum_{n=1}^{N_{0}} a_{n} n^{-s}+S_{K}(s)+S_{K}(1-s) \\
& \quad+C_{K}^{1-s} \Gamma\left(\frac{1-s}{2}\right)^{r_{1}} \Gamma(1-s)^{r_{2}} \sum_{n=1}^{N_{0}} a_{n} n^{s-1}+\epsilon, \text { where } \\
& S_{K}(s)=-\sum_{k=0}^{r_{1}+r_{2}-1} \sum_{i=0}^{i_{0}} \frac{c_{i, k}}{(s+i)^{k+1}}, \\
& c_{i, k}=\sum_{n=1}^{N_{0}} \sum_{j=1}^{r_{1}+r_{2}-k} a_{n} A_{i, j+k}\left(\frac{n}{C_{K}}\right)^{i} \frac{\left(\ln \frac{C_{K}}{n}\right)^{j-1}}{(j-1) !} .
\end{aligned}
$$

Proof. Using formulas (3) and (5), we obtain immediately (14) with

$$
S_{K}(s)=\sum_{n=1}^{N_{0}} a_{n} \sum_{j=1}^{r_{1}+r_{2}} \sum_{i=0}^{i_{0}} A_{i, j}(s)\left(\frac{n}{C_{K}}\right)^{i} \frac{\left(\ln \frac{C_{K}}{n}\right)^{j-1}}{(j-1) !} .
$$

If $s$ is not an integer, $A_{i, j}(s)$ is given by the first line of (13). Rewriting the recurrence, we easily obtain

$$
A_{i, j}(s)=-\sum_{k=0}^{r_{1}+r_{2}-j} \frac{A_{i, j+k}}{(s+i)^{k+1}}
$$

and so

$$
\begin{aligned}
S_{K}(s) & =-\sum_{n=1}^{N_{0}} \sum_{j=1}^{r_{1}+r_{2}} \sum_{i=0}^{i_{0}} \sum_{k=0}^{r_{1}+r_{2}-j} a_{n} \frac{A_{i, j+k}}{(s+i)^{k+1}}\left(\frac{n}{C_{K}}\right)^{i} \frac{\left(\ln \frac{C_{K}}{n}\right)^{j-1}}{(j-1) !} \\
& =-\sum_{k=0}^{r_{1}+r_{2}-1} \sum_{n=1}^{N_{0}} \sum_{i=0}^{i_{0}} \sum_{j=1}^{r_{1}+r_{2}-k} a_{n} \frac{A_{i, j+k}}{(s+i)^{k+1}}\left(\frac{n}{C_{K}}\right)^{i} \frac{\left(\ln \frac{C_{K}}{n}\right)^{j-1}}{(j-1) !}
\end{aligned}
$$

which proves the proposition.

Example. If we define $K$ by the monic polynomial $X^{3}+X^{2}-2 X-1$ and we want 30 digits of accuracy for values of $\zeta_{K}$ near $s=2$, we obtain $N_{0}=134$ and $i_{0}=164$. The number of operations for each value of $s$ is around 132000 with the first form of the method, and only 1100 with the modified form!

As a conclusion, we give another proposition which is almost the same as the preceding one, except that $s$ is now an integer. The proof is the same as that of Proposition 2.2.

Proposition 2.2'. If $s$ is a negative integer and $s \geq-i_{0}$, (if $s$ is positive and $s \leq i_{0}+1$, we take $1-s$ because of the functional equation $\Lambda(s)=\Lambda(1-s)$ ), we have

$$
\begin{aligned}
\Lambda_{K}(s)= & \frac{2^{r_{1}} h_{K} R_{K}}{\omega_{K}(s-1) s}+S_{K}^{\prime}(s) \\
& +C_{K}^{1-s} \Gamma\left(\frac{1-s}{2}\right)^{r_{1}} \Gamma(1-s)^{r_{2}} \sum_{n=1}^{N_{0}} a_{n} n^{s-1}+S_{K}(1-s)+\epsilon
\end{aligned}
$$


where

$$
\begin{aligned}
& S_{K}^{\prime}(s)=-\sum_{k=0}^{r_{1}+r_{2}-1} \sum_{\substack{i=0 \\
i \neq-s}}^{i_{0}} \frac{c_{i, k}}{(s+i)^{k+1}}+c_{-s}^{\prime}, \\
& c_{-s}^{\prime}=C_{K}^{s} \sum_{n=1}^{N_{0}} n^{-s} \sum_{j=1}^{r_{1}+r_{2}+1} A_{-s, j-1} a_{n} \frac{\left(\ln \frac{C_{K}}{n}\right)^{j-1}}{(j-1) !}
\end{aligned}
$$

and $\epsilon$ is the error made truncating the series (3) and (5) to $N_{0}$ and $i_{0}$ terms.

\section{Estimate OF ERROR TERMS}

In this section, we bound the error terms by computing an asymptotic bound for the function $f(x, s)$, and we compute $i_{0}, N_{0}$ and the maximal number of ideals of a given norm so as to guarantee a given accuracy in the computation of $\zeta_{K}(s)$.

3.1. Asymptotic bound for $f(x, s)$. B. L. J. Braaksma explains (see [3], Sections 2,3 and 4) how we can obtain an asymptotic expansion for a class of Barnesintegrals, and thus for $f(x, s)$. We use here his results to obtain an asymptotic bound for $f(x, s)$. Using the notations of [3], (2.25) we have

$$
\begin{aligned}
f(x, s) & =\frac{1}{2 i \pi} \int_{-\delta-i \infty}^{-\delta+i \infty} x^{-z} \Gamma\left(\frac{-z}{2}\right)^{r_{1}} \Gamma(-z)^{r_{2}} \frac{d z}{-z-s} \\
& =\frac{1}{2 i \pi} \int_{-\delta-i \infty}^{-\delta+i \infty} x^{-z} h_{2}(z, s) d z=H_{0}\left(\frac{1}{x}, s\right) .
\end{aligned}
$$

We need an asymptotic bound for $H_{0}\left(\frac{1}{x}, s\right)$ when $x \rightarrow 0^{+}$. We have obtained the following result.

Proposition 3.1. If $0<x \leq 1$, $|\operatorname{Im} s| \leq T$, and $|\operatorname{Re} s| \leq L$, we have:

$$
|f(x, s)| \leq A x^{\frac{r_{1}+r_{2}-3}{N}} \exp \left(-2^{\frac{r_{1}}{N}-1} N x^{-\frac{2}{N}}\right)
$$

with

$$
A=\pi^{\frac{r_{1}+r_{2}}{2}} 2^{\frac{N}{2}} N^{r_{1}+r_{2}-\frac{1}{2}}\left(r_{2}+L N+1\right)^{\frac{r_{2}+L N+1}{2}}(T+L+1)
$$

Proof. We first apply the Euler-Maclaurin formula to obtain an estimate for $h_{2}(z, s)$. We assume in the entire proof that $s$ is a complex number with $|\operatorname{Im} s| \leq T$ and $|\operatorname{Re} s| \leq L$. We let $\mu=N / 2, \beta=2^{r_{1} / 2}$ and $\alpha=\left(r_{1}+r_{2}+3\right) / 2$. We introduce the function

$$
g(z, s)=\frac{(2 \pi)^{1-r_{1}-r_{2}} h_{2}(z, s)\left(\beta \mu^{\mu}\right)^{-z}}{\Gamma(1-\alpha-\mu z)} .
$$

We start with the following formula ([14], p 208, formula (17)):

$$
\ln \Gamma(z+a)=(z+a) \ln z-z+\frac{1}{2} \ln 2 \pi+\phi(z)
$$

with

$$
\phi(z)=\frac{Q(z)}{12 z} \quad \text { and }|Q(z)| \leq 2 \text { if } \operatorname{Re} z>0
$$


We use this result to compute

$$
\begin{aligned}
\ln g(z, s)= & -z \ln \left(\beta \mu^{\mu}\right)-\ln (-s-z)+\left(1-r_{1}-r_{2}\right) \ln 2 \pi \\
& -\left[\left(-\mu z+\frac{1}{2}-\alpha\right) \ln (-\mu z)+\mu z+\frac{1}{2} \ln 2 \pi+\phi\left(-\frac{\mu z}{2}\right)\right] \\
& +r_{1}\left[\left(\frac{-z-1}{2}\right) \ln \left(-\frac{z}{2}\right)+\frac{z}{2}+\frac{1}{2} \ln 2 \pi+\phi\left(-\frac{z}{2}\right)\right] \\
& +r_{2}\left[\left(-z-\frac{1}{2}\right) \ln (-z)+z+\frac{1}{2} \ln 2 \pi+\phi(-z)\right]
\end{aligned}
$$

and we finally have

$$
\ln g(z, s)=c_{0}+\ln \left(\frac{z}{z+s}\right)+\theta(z)
$$

with

$$
|\theta(z)| \leq \frac{\left(\frac{2}{\mu}+2 r_{1}+r_{2}\right)}{6|z|} \leq \frac{\left(\frac{2}{\mu}+2 r_{1}+r_{2}\right)}{6(L+1)}
$$

because we can assume that $|z| \geq L+1$ (we take $\delta=L+1$ in the integral defining $f(x, s)$ ). We now bound the modulus of the logarithm. We have

$$
\left|\ln \left(\frac{z}{z+s}\right)\right| \leq \sqrt{\ln ^{2} \frac{|z|}{|z+s|}+\pi^{2}} \leq 4+\ln (T+L+1) \quad \text { if }|z| \leq 3|s|
$$

since $\operatorname{Re} z=-L-1,|\operatorname{Re} s| \leq L$, and $|\operatorname{Im} s| \leq T$. We also have

$$
\left|\ln \left(\frac{z}{z+s}\right)\right|=\left|\ln \left(1-\frac{s}{z+s}\right)\right| \leq \sum_{n \geq 1} \frac{|s|^{n}}{n|z+s|^{n}} \leq \ln 2 \quad \text { if }|z|>3|s|
$$

and we finally obtain

$$
|g(z, s)| \leq \exp \left[c_{0}+\frac{1}{6(L+1)}\left(2 r_{1}+r_{2}+\frac{2}{\mu}\right)+4+\ln (T+L+1)\right]=\gamma .
$$

As in [3] we can say that

$$
h_{2}(z, s)=\rho_{0}(z)\left(\beta \mu^{\mu}\right)^{z} \Gamma(-\mu z+1-\alpha)(2 \pi)^{r_{1}+r_{2}-1} \quad \text { with }\left|\rho_{0}(z)\right| \leq \gamma
$$

and that

$$
\begin{array}{r}
f\left(\frac{x}{\beta \mu^{\mu}}, s\right)=-i(2 \pi)^{r_{1}+r_{2}-2} \int_{-L-1-i \infty}^{-L-1+i \infty} x^{-z} \rho_{0}(z) \Gamma(1-\mu z-\alpha) d z \\
\text { with }\left|\rho_{0}(z)\right| \leq \gamma .
\end{array}
$$

We use now the same principle as Braaksma introducing the function $\sigma(x)$.

$$
\begin{aligned}
\sigma(x) & =\int_{-L-1-i \infty}^{-L-1+i \infty} x^{-z} \rho_{0}(z) \Gamma(1-\mu z-\alpha) d z \\
& =\int_{-L-1-i \infty}^{-L-1+i \infty} x^{-z} \rho_{0}(z) \frac{\Gamma(3-\mu z-\alpha)}{(1-\mu z-\alpha)(2-\mu z-\alpha)} d z .
\end{aligned}
$$

We start with the formula

$$
\Gamma(3-\mu z-\alpha) x^{-z}=x^{\frac{\alpha-3}{\mu}} \int_{0}^{+\infty} t^{2-\mu z-\alpha} \exp \left[-x^{-\frac{1}{\mu} t}\right] d t
$$


with Re $z=-L-1$ (In [3], Braaksma shows that the integral is absolutely convergent.) Using now this relation, we easily obtain (see [3] for a rigorous justification)

$$
\begin{aligned}
& \sigma(x)=x^{\frac{\alpha-3}{\mu}} \int_{0}^{+\infty} \rho(t) \exp \left[-x^{-\frac{1}{\mu} t}\right] d t \\
& \text { with } \rho(t)=\int_{-L-1-i \infty}^{-L-1+i \infty} \rho_{0}(z) t^{2-\mu z-\alpha} \frac{d z}{(1-\mu z-\alpha)(2-\mu z-\alpha)} .
\end{aligned}
$$

We have now

$$
\begin{aligned}
& |\rho(t)| \leq t^{2+(L+1) \mu-\alpha} \int_{-L-1-i \infty}^{-L-1+i \infty}\left|\rho_{0}(z)\right| \frac{|d z|}{|\alpha+\mu z-1||\alpha+\mu z-2|} \\
& \quad \leq \gamma t^{2+(L+1) \mu-\alpha} \int_{-\infty}^{+\infty} \frac{d \theta}{\sqrt{\left[(\alpha-(L+1) \mu-1)^{2}+\mu^{2} \theta^{2}\right]\left[(\alpha-(L+1) \mu-2)^{2}+\mu^{2} \theta^{2}\right]}} \\
& \quad \leq \gamma K t^{2+(L+1) \mu-\alpha}
\end{aligned}
$$

where

$$
\begin{aligned}
K & \leq \int_{-\infty}^{-1} \frac{d \theta}{\mu^{2} \theta^{2}}+\int_{1}^{+\infty} \frac{d \theta}{\mu^{2} \theta^{2}}+\int_{-1}^{1} \frac{d \theta}{|(L+1) \mu+1-\alpha||(L+1) \mu+2-\alpha|} \\
& \leq \frac{2}{\mu^{2}}+\frac{1}{[(L+1) \mu+1-\alpha]^{2}} .
\end{aligned}
$$

So we have proved that $|\rho(t)| \leq K \gamma t^{2+(L+1) \mu-\alpha}$ for $t>0$. Moreover we know that $\rho(t)=0$ when $t \in] 0,1]$ (see [3]). From this and the precedent formulas we derive

$$
\begin{aligned}
|\sigma(x)| & \leq K \gamma x^{\frac{\alpha-3}{\mu}} \int_{1}^{+\infty} t^{2+(L+1) \mu-\alpha} \exp \left[-t x^{-\frac{1}{\mu}}\right] d t \\
& \leq K \gamma x^{\frac{\alpha-3}{\mu}} \exp \left[-x^{-\frac{1}{\mu}}\right] \int_{1}^{+\infty} t^{2+(L+1) \mu-\alpha} \exp [-(t-1) \eta] d t
\end{aligned}
$$

where $\eta$ is an arbitrary real number and $x$ is such that $x^{-\frac{1}{\mu}} \geq \eta$. We take $\eta=1$ (and thus $x \leq 1$ ) and we use a rough estimate for

$$
I=\int_{1}^{+\infty} t^{2+(L+1) \mu-\alpha} \exp [-(t-1) \eta] d t
$$

to obtain that

$$
I \leq K^{\prime}=2(4+(2 L+2) \mu-2 \alpha)^{2+(L+1) \mu-\alpha} \exp \left[\alpha-\frac{3}{2}-(L+1) \mu\right] .
$$

From all the preceding relations and with a little computation we obtain

$$
|\sigma(x)| \leq K \gamma K^{\prime} x^{\frac{\alpha-3}{\mu}} \exp \left[-x^{\frac{1}{\mu}}\right] \quad \text { if } 0<x \leq 1,|\operatorname{Im} s| \leq T \text {, and }|\operatorname{Re} s| \leq L
$$

and finally

$$
|f(x, s)| \leq\left[K \gamma K^{\prime}(2 \pi)^{r_{1}+r_{2}-2}\left(\beta \mu^{\mu}\right)^{\frac{\alpha-3}{\mu}}\right] x^{\frac{\alpha-3}{\mu}} \exp \left[-\beta^{\frac{1}{\mu}} \mu x^{-\frac{1}{\mu}}\right]
$$

if $0<x \leq 1,|\operatorname{Im} s| \leq T$, and $|\operatorname{Re} s| \leq L$. 
3.2. The bound $N_{0}$. Suppose we want to compute a value of $\zeta_{K}$ with an absolute error $\epsilon$. We have to evaluate the error that we make when truncating the series in (3) and then to compute the bound $N_{0}$ depending on $\epsilon$. The problem is to find the "best" value of $N_{0}$ such that

$$
\sum_{n>N_{0}}|r(n, s)|=\sum_{n>N_{0}} a_{n}\left|f\left(\frac{C_{K}}{n}, s\right)+f\left(\frac{C_{K}}{n}, 1-s\right)\right| \leq \epsilon .
$$

We define the coefficients $d_{N}(n)$ by $\zeta(s)^{N}=\sum_{n \geq 1} d_{N}(n) n^{-s}$ and using Theorem 2 in [5], we obtain the inequality

$$
a_{n} \leq d_{N}(n) \leq n^{a} \quad \text { where } a=\frac{(\ln N)^{3}}{\ln 2}
$$

From this and (17) we can derive

$$
|r(n, s)| \leq 2 A C_{K}^{\frac{\alpha-3}{\mu}} n^{a+\frac{3-\alpha}{\mu}} \exp \left[\frac{-n^{\frac{2}{N}}}{C_{K}^{\frac{2}{N}}}\right] \leq C n^{\delta} \exp \left[\frac{-n^{\frac{2}{N}}}{b}\right]
$$

and

$$
R\left(N_{0}, s\right)=\sum_{n>N_{0}}|r(n, s)| \leq C \sum_{n>N_{0}} n^{\delta} \exp \left[\frac{-n^{\frac{2}{N}}}{b}\right] \leq C \int_{N_{0}}^{+\infty} t^{\delta} \exp \left[\frac{-t^{\frac{2}{N}}}{b}\right] d t
$$

We use a rough estimate for this last integral and we obtain

Proposition 3.3. $N_{0}$ is the smallest integer such that

$$
N_{0} \geq C_{K}\left(2 \ln \frac{K_{0}}{\epsilon}\right)^{\frac{N}{2}}
$$

where $K_{0}=C \frac{N b^{\frac{(\delta+1) N}{2}}}{2}\left[\frac{(\delta+1) N-2}{e}\right]^{\frac{(\delta+1) N}{2}-1}$.

3.3. The bound $i_{0}$. We want now to estimate the error that we make when we compute values of the function $f(x, s)$. Let $\epsilon^{\prime}$ be the absolute precision that we want to obtain when we compute a single value of $f(x, s)$. Since we compute $N_{0}$ successive values of this function, it is clear that $\epsilon^{\prime}=\epsilon / N_{0}$. We prove the following result:

Proposition 3.3. $i_{0}$ is the smallest integer such that

$$
\left(\frac{C_{K}}{N_{0}}\right)^{i_{0}+\frac{1}{2}}\left(\left\lfloor\frac{i_{0}}{2}\right\rfloor !\right)^{r_{1}}\left(i_{0} !\right)^{r_{2}} \geq \frac{(2 \sqrt{\pi})^{r_{2}} 5^{r_{1}}}{\pi^{\frac{3}{2}} \epsilon^{\prime}} .
$$

Proof. We estimate the remainder $r\left(i_{0}, s\right)$ of the series in formula (5) when we truncate this series to $i_{0}$ terms. In fact, $r\left(i_{0}, s\right)$ depends also on $x$ but we can easily see that the convergence of the series becomes slower when $x$ decreases. So we set $x=C_{K} / N_{0}$ which is the smallest value of $x$, and we compute the corresponding remainder. We easily have

$$
\begin{aligned}
\left|r\left(i_{0}, s\right)\right| & \leq \frac{1}{2 \pi} \int_{-i_{0}-\frac{1}{2}-i \infty}^{-i_{0}-\frac{1}{2}+i \infty}\left|\left(\frac{C_{K}}{N_{0}}\right)^{z}\right|\left|\Gamma\left(\frac{z}{2}\right)\right|^{r_{1}}|\Gamma(z)|^{r_{2}} \frac{d z}{z-s} \\
& \leq \int_{-\infty}^{\infty}\left(\frac{C_{K}}{N_{0}}\right)^{-i_{0}-\frac{1}{2}}\left|\Gamma\left(\frac{-i_{0}-\frac{1}{2}+i t}{2}\right)\right|^{r_{1}}\left|\Gamma\left(-i_{0}-\frac{1}{2}+i t\right)\right|^{r_{2}} d t .
\end{aligned}
$$


As in [8], we have

$$
\begin{aligned}
\left|\Gamma\left(-i_{0}-\frac{1}{2}+i t\right)\right| & \leq \frac{\left|\Gamma\left(\frac{1}{2}+i t\right)\right|}{\left|\left(-i_{0}-\frac{1}{2}+i t\right)\left(-i_{0}+\frac{1}{2}+i t\right) \ldots\left(-\frac{1}{2}+i t\right)\right|} \\
& \leq \frac{2}{i_{0} !}\left|\Gamma\left(\frac{1}{2}+i t\right)\right|
\end{aligned}
$$

and

$$
\left|\Gamma\left(\frac{-i_{0}-\frac{1}{2}+i t}{2}\right)\right| \leq \frac{4}{\left[\frac{i_{0}}{2}\right\rfloor !}\left|\Gamma\left(\frac{3}{4}+\frac{i t}{2}\right)\right|
$$

and combining these inequalities with the first one, we obtain formula (20).

3.4. Conclusion. We are now able to write a complete algorithm for computing values of $\Lambda_{K}$ (and $\zeta_{K}$ ) in the complex plane.

In the first part of the algorithm, we compute all the coefficients and data which depend only on the number field and on the absolute precision $\epsilon$. We use the algorithms implemented in Pari to compute the regulator and the class number. This part of the program requires a few minutes of CPU time.

In the second part, we only make basic operations (see formulas (14) and (15)) which require only a few seconds of CPU time.

The problem with this method is that we stop our computations when the remainder is smaller than the absolute precision required. So, if the value of $\Lambda_{K}$ is very small (if $s$ is far from the real axis), we obtain few significant digits. The only solution that we have found is to increase this number of digits with the height of our computations (see Section 5 for more details).

The following table shows the execution time of the program in various cases (the smallest and largest number field of Tables 6.1 to 6.7). The third column contains the execution time of the initialisation part. The time needed to compute a real or a complex value of $\zeta_{K}(s)$ is given in columns 5 and 6 . All these times are given in seconds.

\begin{tabular}{|l|c|c|c|c|}
\hline \multicolumn{1}{|c|}{$P(x)$} & Digits & Init & Real & Cplex \\
\hline$x^{3}+x^{2}-1$ & 30 & 5 & 0.2 & 0.5 \\
$x^{3}+x^{2}-1$ & 120 & 280 & 5 & 12 \\
$x^{3}+x^{2}-10 x-8$ & 30 & 11 & 0.3 & 0.8 \\
$x^{3}+x^{2}-10 x-8$ & 120 & 1517 & 11 & 25 \\
$x^{4}+x^{3}-x^{2}-x+1$ & 35 & 17 & 0.4 & 1 \\
$x^{4}+x^{3}-x^{2}-x+1$ & 90 & 493 & 5 & 12 \\
$x^{4}+2 x^{3}-3 x^{2}-2 x+1$ & 35 & 65 & 1 & 2.3 \\
$x^{4}+2 x^{3}-3 x^{2}-2 x+1$ & 90 & 2773 & 18 & 40 \\
\hline
\end{tabular}

\section{The generalized Turing Criterion}

In this section, we generalize Turing's criterion to Dedekind zeta functions. The principle is to combine a count of the zeros of $\zeta_{k}(s)$ in a segment $\left[\frac{1}{2}, \frac{1}{2}+i T\right]$, together with the existence of sufficiently long intervals where the zeros are fairly regularly spaced, to rigorously prove that all zeros with imaginary part $t \in[0, T]$ are on the critical line. 
Here are our principal references for this section. The first one is [21] (in 1953) where Turing explains his method. Unfortunately, there are many mistakes in this paper but Lehman gives a corrected version of Turing's original proof in [12]. Finally, we refer to a third paper: in [20], Rumely generalizes the criterion to Dirichlet L-series.

We introduce the function

$$
S_{K}(t)=\frac{1}{\pi} \arg \zeta_{K}\left(\frac{1}{2}+i t\right)
$$

where the value of the argument is obtained, if $t$ is not the ordinate of a zero, by continuous variation along the line from $\infty+i t$ to $\frac{1}{2}+i t$ starting with the value zero. Let now $S_{K}^{1}(t)$ be

$$
S_{K}^{1}(t)=\int_{0}^{t} S_{K}(u) d u
$$

We begin this section by one of the two main theorems.

4.1. The main result about $S_{K}^{1}$. This first theorem gives us an estimate for the function $S_{K}^{1}$. The proof is rather long but is similar to the one in [20], p 427-433. Of course, the result depends on the degree, the signature and the discriminant of the number field.

Theorem 4.1. If $t_{1}$ and $t_{2}$ are real numbers such that $40<t_{1}<t_{2}$, we have

$$
\left|S_{K}^{1}\left(t_{2}\right)-S_{K}^{1}\left(t_{1}\right)\right| \leq(0.2627+1.8392 N)+0.122 \ln \left[D_{K}\left(\frac{t_{2}}{2 \pi}\right)^{N}\right] .
$$

Proof. We assume that $t_{1}>40$ and $t_{2}>40$ since in the case of number fields of degree 4 , we compute zeros to height 40 . We note that there is a constant term (0.2627) which is independent of the number field. This comes from a result of Rademacher used in the proof of Theorem 4.2. We start the proof by writing the integral as follows.

Lemma 4.1. If $t_{1}$ and $t_{2}$ are not the ordinate of a zero and $40<t_{1}<t_{2}$, we have

$$
\pi\left|S_{K}^{1}\left(t_{2}\right)-S_{K}^{1}\left(t_{1}\right)\right|=\int_{\frac{1}{2}+i t_{2}}^{+\infty+i t_{2}} \ln \left|\zeta_{K}(s)\right| d s-\int_{\frac{1}{2}+i t_{1}}^{+\infty+i t_{1}} \ln \left|\zeta_{K}(s)\right| d s
$$

Proof. This result is an application of Cauchy's theorem (see Lemma 1, p. 106 in [21] or [13], p. 534).

We have now to bound the integrals that we obtain in formula (22). We begin with a first inequality.

Theorem 4.2. If $t>40$ we have

$$
\begin{aligned}
I_{K}(t) & =\int_{\frac{1}{2}+i t}^{+\infty+i t} \ln \left|\zeta_{K}(s)\right| d s \\
& \leq(0.8252+2.329 N)+0.1407 \ln \left[\left|D_{K}\right|\left(\frac{t}{2 \pi}\right)^{N}\right] .
\end{aligned}
$$

Proof. The proof of this theorem is quite easy. We apply the following result from Rademacher (see [18] p. 200). 
Lemma 4.2. If $0<\eta<\frac{1}{2}$ and $s=\sigma+i$, we have with the usual notations

$$
\begin{aligned}
& \left|\zeta_{K}(s)\right| \leq 3\left|\frac{1+s}{1-s}\right| \zeta(1+\eta)^{N}\left[\left|D_{K}\right|\left(\frac{|1+s|}{2 \pi}\right)^{N}\right]^{\frac{1+\eta-\sigma}{2}} \\
& \text { for }-\eta \leq \sigma \leq 1+\eta
\end{aligned}
$$

The proof is given in [18]. We apply this result, for $\eta=\frac{1}{4}$ and we use Lemma 3 , p. 305 in [12]. We have

$$
\begin{aligned}
\ln \left|\zeta_{K}(s)\right| \leq & \ln 3+\ln \left|\frac{1+s}{1-s}\right| \\
& +\left(\frac{5}{8}-\frac{\sigma}{2}\right) \ln \left[\left|D_{K}\right|\left(\frac{|1+s|}{2 \pi}\right)^{N}\right]+N \ln \zeta\left(\frac{5}{4}\right) .
\end{aligned}
$$

- If $t>40$ and $\frac{1}{2} \leq \sigma \leq \frac{5}{4}$, we have

$$
\begin{aligned}
& \ln \left|\frac{1+s}{1-s}\right| \leq 0.00156 \\
& \text { and } \ln \left(\frac{|1+s|}{t}\right) \leq 0.00158 \quad \Rightarrow \quad \ln |1+s|^{N} \leq \ln t^{N}+0.00158 N
\end{aligned}
$$

so

$$
\left(\frac{5}{8}-\frac{\sigma}{2}\right) \ln \left[\left|D_{K}\right|\left(\frac{|1+s|}{2 \pi}\right)^{N}\right] \leq\left(\frac{5}{8}-\frac{\sigma}{2}\right)\left(\ln \left[\left|D_{K}\right|\left(\frac{t}{2 \pi}\right)^{N}\right]+0.00158 N\right) .
$$

- $N \ln \zeta\left(\frac{5}{4}\right) \leq 1.525 N$.

We combine these results with formula $\left(24^{\prime}\right)$ and we obtain

$$
\begin{aligned}
\int_{\frac{1}{2}+i t}^{+\infty+i t} \ln \left|\zeta_{K}(s)\right| d s \leq & \int_{\frac{1}{2}}^{\frac{5}{4}}\left[\left(\frac{5}{8}-\frac{\sigma}{2}\right)\left(\ln \left[\left|D_{K}\right|\left(\frac{t}{2 \pi}\right)^{N}\right]+0.0016 N\right)\right] d \sigma \\
& +\frac{3}{4}(\ln 3+0.0016+1.525 N)+\int_{\frac{5}{4}}^{+\infty} \ln \left|\zeta_{K}(\sigma+i t)\right| d \sigma \\
\leq & 0.1407 \ln \left[\left|D_{K}\right|\left(\frac{t}{2 \pi}\right)^{N}\right]+0.00023 N+0.824 \\
& +0.0012+1.1438 N+1.184 N \quad(*) \\
\leq & (0.8252+2.329 N)+0.1407 \ln \left[\left|D_{K}\right|\left(\frac{t}{2 \pi}\right)^{N}\right] .
\end{aligned}
$$

For $\left(^{*}\right)$, note that $\ln \left|\zeta_{K}(\sigma+i t)\right| \leq N \ln |\zeta(\sigma)|$, and use again Lemma 3' of [12].

We now prove another inequality about the integral $I_{K}(t)$.

Theorem 4.3. If $t>40$, we have

$$
I_{K}(t)=\int_{\frac{1}{2}+i t}^{+\infty+i t} \ln \left|\zeta_{K}(s)\right| d s \geq-3.4489 N-0.24 \ln \left[\left|D_{K}\right|\left(\frac{t}{2 \pi}\right)^{N}\right] .
$$


Proof. We start writing the integral in the following form

$$
\begin{aligned}
\int_{\frac{1}{2}+i t}^{+\infty+i t} \ln \left|\zeta_{K}(s)\right| d s= & \int_{\frac{1}{2}+i t}^{+\infty+i t} \ln \left|\frac{\zeta_{K}(s) \zeta_{K}(s+2)}{\zeta_{K}(s+1)^{2}}\right| d s+\int_{\frac{3}{2}+i t}^{+\infty+i t} \ln \left|\zeta_{K}(s)\right| d s \\
& +\int_{\frac{3}{2}+i t}^{\frac{5}{2}+i t} \ln \left|\zeta_{K}(s)\right| d s=I_{1}+I_{2}+I_{3} .
\end{aligned}
$$

1) Estimate for the integrals $I_{2}$ and $I_{3}$. If we set $s=\sigma+i t$, we have $\sigma>1$, so

$$
\begin{aligned}
& \ln \left|\zeta_{K}(s)\right|=-\sum_{\mathfrak{p}} \ln \left|1-p^{-f_{\mathfrak{p}} s}\right| \\
& \left|1-p^{-f_{\mathfrak{p}} s}\right| \leq 1+p^{-f_{\mathfrak{p}} \sigma} \leq 1+p^{-\sigma} \\
& \text { so } \quad \ln \left|1-p^{-f_{\mathfrak{p}} s}\right| \leq \ln \left(1-p^{-2 \sigma}\right)-\ln \left(1-p^{-\sigma}\right)
\end{aligned}
$$

and we find

$$
\ln \left|\zeta_{K}(s)\right| \geq N(\ln |\zeta(2 \sigma)|-\ln |\zeta(\sigma)|) .
$$

We use now the inequalities from [20], p. 431,

$$
\begin{array}{ll}
\int_{\frac{3}{2}}^{\frac{5}{2}} \ln |\zeta(\sigma)| d \sigma<0.5382 & \int_{\frac{5}{2}}^{+\infty} \ln |\zeta(\sigma)| d \sigma<0.3445, \\
\int_{5}^{\infty} \ln |\zeta(\sigma)| d \sigma<0.0495 & \int_{3}^{+\infty} \ln |\zeta(\sigma)| d \sigma<0.1779
\end{array}
$$

and combining these with inequality (26), we easily find the required estimate:

$$
I_{2}+I_{3} \geq-1.2183 N \text {. }
$$

2) Estimate for the integral $I_{1}$. We use exactly the same principle as Rumely in [20] (formula (16), p. 431), to prove the following inequality:

$$
\int_{\frac{1}{2}+i t}^{+\infty+i t} \ln \left|\zeta_{K}(s)\right| d s \geq \int_{\frac{1}{2}+i t}^{\frac{3}{2}+i t} \ln \left|\frac{\zeta_{K}(s)}{\zeta_{K}(s+1)}\right| d s-1.2183 N .
$$

We apply the following result about $\Lambda_{K}$ (see [11]).

Lemma 4.3. The function $s \rightarrow s(s-1) \Lambda_{K}(s)$ is holomorphic on the complex plane and its zeros are the non-trivial zeros $\rho$ of the Dedekind zeta function $\zeta_{K}$. So $\Lambda_{K}$ can be expanded in a Weierstrass-Hadamard product

$$
s(s-1) \Lambda_{K}(s)=\exp (a+b s) \prod_{\rho}\left(1-\frac{s}{\rho}\right) \exp \left(\frac{s}{\rho}\right) \quad \text { with } \operatorname{Re} b=-\sum_{\rho} \operatorname{Re} \frac{1}{\rho} .
$$

In our special case, if $C_{K}$ is defined as usual, we have

$$
\frac{(s-1) \Gamma\left(\frac{s}{2}\right)^{r_{1}} \Gamma(s)^{r_{2}} \zeta_{K}(s)}{(s+1) C_{K} \Gamma\left(\frac{s+1}{2}\right)^{r_{1}} \Gamma(s+1)^{r_{2}} \zeta_{K}(s+1)}=\exp (-b) \prod_{\rho}\left(\frac{1-\frac{s}{\rho}}{1-\frac{s+1}{\rho}}\right) \exp \left(\frac{-1}{\rho}\right) .
$$


Combining this identity with (27) we obtain

$$
\begin{aligned}
\ln \left|\frac{\zeta_{K}(s)}{\zeta_{K}(s+1)}\right|= & -\operatorname{Re} b+\ln \left|\frac{s+1}{s-1}\right|+\ln C_{K}+\ln \left|\frac{\Gamma\left(\frac{s+1}{2}\right)^{r_{1}} \Gamma(s+1)^{r_{2}}}{\Gamma\left(\frac{s}{2}\right)^{r_{1}} \Gamma(s)^{r_{2}}}\right| \\
& +\sum_{\rho} \ln \left|\frac{1-\frac{s}{\rho}}{1-\frac{s+1}{\rho}}\right|-\operatorname{Re} \frac{1}{\rho} \\
\geq & \ln C_{K}+\ln \left|\frac{\Gamma\left(\frac{s+1}{2}\right)^{r_{1}} \Gamma(s+1)^{r_{2}}}{\Gamma\left(\frac{s}{2}\right)^{r_{1}} \Gamma(s)^{r_{2}}}\right|+\sum_{\rho} \ln \left|\frac{1-\frac{s}{\rho}}{1-\frac{s+1}{\rho}}\right| .
\end{aligned}
$$

We have thus proved the inequality

$$
\begin{aligned}
\int_{\frac{1}{2}+i t}^{+\infty+i t} \ln \left|\zeta_{K}(s)\right| d s \geq & \ln C_{K}+r_{1} \int_{\frac{1}{2}+i t}^{\frac{3}{2}+i t} \ln \left|\frac{\Gamma\left(\frac{s+1}{2}\right)}{\Gamma\left(\frac{s}{2}\right)}\right| d s \\
& +r_{2} \int_{\frac{1}{2}+i t}^{\frac{3}{2}+i t} \ln \left|\frac{\Gamma(s+1)}{\Gamma(s)}\right| d s \\
& +\int_{\frac{1}{2}+i t}^{\frac{3}{2}+i t} \sum_{\rho} \ln \left|\frac{1-\frac{s}{\rho}}{1-\frac{s+1}{\rho}}\right| d s-1.2183 N \\
\geq & \ln C_{K}+H+I+J-1.2183 N
\end{aligned}
$$

2.1) Estimate for the integrals $H$ and $I$. We apply the mean value theorem to estimate the integrals $H$ and $I$ (for details, see [12], p. 311). We obtain

$$
\begin{aligned}
& H=\frac{r_{1}}{2} \operatorname{Re} \frac{\Gamma^{\prime}}{\Gamma}\left(\frac{i t}{2}+\tau\right) \quad \text { where } \frac{1}{4}<\tau<\frac{5}{4}, \\
& I=r_{2} \operatorname{Re} \frac{\Gamma^{\prime}}{\Gamma}(i t+\tau) \quad \text { where } \frac{1}{2}<\tau<\frac{5}{2},
\end{aligned}
$$

and we use the following result about $\frac{\Gamma^{\prime}}{\Gamma}$ (see [12], p. 308, Lemma 8)

$$
\frac{\Gamma^{\prime}}{\Gamma}(z)=\ln z-\frac{1}{2 z}+\Theta\left(\frac{2}{\pi^{2}\left|\operatorname{Im}^{2} z-\operatorname{Re}^{2} z\right|}\right) \quad \text { if } \operatorname{Re} z>0
$$

where the notation $f=\Theta(g)$ means $-g \leq f \leq g$. We obtain

$$
H \geq \frac{r_{1}}{2}\left(\ln \frac{t}{2}-0.0021\right) \quad \text { and } \quad I \geq r_{2}(\ln t-0.001)
$$

and from these inequalities we derive

$$
\begin{aligned}
\int_{\frac{1}{2}+i t}^{+\infty+i t} \ln \left|\zeta_{K}(s)\right| d s \geq & \ln C_{K}+\frac{N}{2} \ln \frac{t}{2}+J-0.0011 r_{1} \\
& +(\ln 2-0.001) r_{2}-1.2183 N
\end{aligned}
$$

2.2) Estimate for the integral $J$.

$$
J=\sum_{\rho} \int_{\frac{1}{2}+i t}^{\frac{3}{2}+i t} \ln \left|\frac{s-\rho}{s+1-\rho}\right| d s \geq-1.48 \sum_{\rho} \operatorname{Re}\left(\frac{1}{\frac{3}{2}+i t-\rho}\right) .
$$

The interchange of summation and integration is justified in [12], p. 311, and see [12], Lemma 7, p. 307 for the last inequality. We now compute the sum 
$\sum_{\rho} \operatorname{Re}(1 /(s-\rho))$. We logarithmically differentiate the Weierstrass-Hadamard product and we obtain

$$
\frac{2 s-1}{s(s-1)}+\frac{\zeta_{K}^{\prime}}{\zeta_{K}}(s)+\frac{r_{1}}{2} \frac{\Gamma^{\prime}}{\Gamma}\left(\frac{s}{2}\right)+r_{2} \frac{\Gamma^{\prime}}{\Gamma}(s)+\ln C_{K}=b+\sum_{\rho}\left(\frac{1}{\rho}-\frac{1}{\rho-s}\right) .
$$

We take real parts, we use the relation $\operatorname{Re} b=-\sum_{\rho} \operatorname{Re} \frac{1}{\rho}$ and we get

$$
\begin{aligned}
\sum_{\rho} \operatorname{Re}\left(\frac{1}{s-\rho}\right)= & \ln C_{K}+\operatorname{Re} \frac{\zeta_{K}^{\prime}}{\zeta_{K}}(s)+\operatorname{Re}\left(\frac{r_{1}}{2} \frac{\Gamma^{\prime}}{\Gamma}\left(\frac{s}{2}\right)+r_{2} \frac{\Gamma^{\prime}}{\Gamma}(s)\right) \\
& +\operatorname{Re}\left(\frac{2 s-1}{s(s-1)}\right)
\end{aligned}
$$

and if $s=\frac{3}{2}+i t$

$$
\begin{aligned}
J \geq-1.48\left[\ln C_{K}+\operatorname{Re} \frac{\zeta_{K}^{\prime}}{\zeta_{K}}\left(\frac{3}{2}+i t\right)\right. & \\
+\operatorname{Re}\left(\frac{r_{1}}{2} \frac{\Gamma^{\prime}}{\Gamma}\left(\frac{3}{4}+\frac{i t}{2}\right)\right. & \left.+r_{2} \frac{\Gamma^{\prime}}{\Gamma}\left(\frac{3}{2}+i t\right)\right) \\
& \left.+2 \operatorname{Re}\left(\frac{1+i t}{\frac{3}{4}-t^{2}+2 i t}\right)\right] .
\end{aligned}
$$

- We easily see that

$$
2 \operatorname{Re}\left(\frac{1+i t}{\frac{3}{4}-t^{2}+2 i t}\right) \leq 0.00125 \quad \text { if } t>40
$$

and that

$$
\operatorname{Re} \frac{\zeta_{K}^{\prime}}{\zeta_{K}}(s) \leq-N \frac{\zeta^{\prime}}{\zeta}(\sigma) \quad \text { where } s=\sigma+i t \text { and } \sigma>1
$$

so we have

$$
\operatorname{Re} \frac{\zeta_{K}^{\prime}}{\zeta_{K}}\left(\frac{3}{2}+i t\right) \leq 1.506 N
$$

- Using (28) once more, one finds

$$
\begin{aligned}
& \frac{r_{1}}{2} \operatorname{Re} \frac{\Gamma^{\prime}}{\Gamma}\left(\frac{3}{4}+\frac{i t}{2}\right) \leq \frac{r_{1}}{2}\left(\ln \frac{t}{2}+0.0008\right) \text { and } \\
& r_{2} \operatorname{Re} \frac{\Gamma^{\prime}}{\Gamma}\left(\frac{3}{2}+i t\right) \leq r_{2}(\ln t+0.0006)
\end{aligned}
$$

so

$$
\begin{aligned}
J \geq & -1.48 \ln C_{K}-2.2289 N-0.74 N \ln \frac{t}{2}-0.0006 r_{1} \\
& -1.48(\ln 2-0.0006) r_{2}+0.00236 .
\end{aligned}
$$

Thus, combining all the preceding results, one gets

$$
\int_{\frac{1}{2}+i t}^{+\infty+i t} \ln \left|\zeta_{K}(s)\right| d s \geq-3.4489 N-0.24 \ln \left[\left|D_{K}\right|\left(\frac{t}{2 \pi}\right)^{N}\right]
$$

and this is the lower bound of Theorem 4.3.

Theorem 4.1 follows immediately from Lemma 4.1, Theorem 4.2 and Theorem 4.3. 
4.2. The argument principle. In this section, we summarize some well-known properties of $\zeta_{K}(s)$ and we start by introducing the following notations:

$$
N_{K}(T) \text { is the number of zeros of } \zeta_{K} \text { of the form } \rho=\sigma+i t, 0 \leq t \leq T \text {, }
$$

$$
\Phi_{K}(t)=\operatorname{Im}\left(r_{1} \ln \Gamma\left(\frac{1}{4}+\frac{i t}{2}\right)+r_{2} \ln \Gamma\left(\frac{1}{2}+i t\right)\right)+t \ln \frac{\sqrt{\left|D_{K}\right|}}{2^{r_{2}} \pi^{\frac{N}{2}}} ;
$$

we then have

$$
\begin{aligned}
& \Lambda_{K}\left(\frac{1}{2}+i t\right)=\exp [f(t)] \exp \left[i \Phi_{K}(t)\right] \zeta_{K}\left(\frac{1}{2}+i t\right), \\
& Z_{K}(t)=\exp \left[i \Phi_{K}(t)\right] \zeta_{K}\left(\frac{1}{2}+i t\right) .
\end{aligned}
$$

We see that $Z_{K}$ takes real values when $t$ is real, and has the same zeros as $\Lambda_{K}$ (which are the non trivial zeros of $\zeta_{K}$ ). Thus, simple zeros of $\zeta_{K}$ on the critical line can be located by finding changes of sign of $Z_{K}(t)$ on a segment $[0, T]$.

Suppose now that we have found $N_{K}^{\prime}(T)$ zeros with this method. We want to compute $N_{K}(T)$, and if $N_{K}(T)=N_{K}^{\prime}(T)$, then GRH holds for the number field $K$ to the height $T$. There is no zero of $\zeta_{K}$ outside of the critical strip $(0 \leq \sigma \leq 1)$ except for the trivial zeros, so $N_{K}(T)$ is the number of zeros in the rectangle $0 \leq t \leq T$ and $0 \leq \sigma \leq 1$. We apply the argument principle to the function $s(s-1) \Lambda_{K}$ which is holomorphic on the complex plane. We define $R$ as the rectangle $\{s \in \mathbb{C} /-\epsilon \leq \operatorname{Re} s \leq 1+\epsilon$ and $0 \leq \operatorname{Im} s \leq T\}$ and $\partial R$ is the boundary of $R$ oriented in the usual counterclockwise direction. It is assumed that $T$ is such that there are no roots $\rho$ on the line $\operatorname{Im} s=T$. We call $C$ the portion of $\partial R$ from $1+\epsilon$ to $\frac{1}{2}+i T$. If $L_{K}(s)=s(s-1) \Lambda_{K}(s)$, we have

$$
\begin{aligned}
N_{K}(T)= & \frac{1}{2 i \pi} \int_{\partial R} \frac{L_{K}^{\prime}}{L_{K}}(s) d s=\frac{1}{\pi} \operatorname{Im}\left(\int_{C} \frac{L_{K}^{\prime}}{L_{K}}(s) d s\right) \\
= & \frac{1}{\pi} \operatorname{Im}\left(\int_{C} \frac{d}{d s}\left[r_{1} \ln \Gamma\left(\frac{s}{2}\right)+r_{2} \ln \Gamma(s)+s \ln C_{K}\right] d s\right) \\
& +\frac{1}{\pi} \operatorname{Im}\left(\int_{C} \frac{d}{d s}[\ln s(s-1)] d s\right)+\frac{1}{\pi} \operatorname{Im}\left(\int_{C} \frac{\zeta_{K}^{\prime}}{\zeta_{K}}(s) d s\right)
\end{aligned}
$$

and we obtain (see [6], p. 128 for a rigorous justification) the relation

$$
N_{K}(T)=\frac{\Phi_{K}(T)}{\pi}+1+S_{K}(T) .
$$

We can easily compute an approximation of $\Phi_{K}$, so the main problem is to estimate the function $S_{K}(t)$.

Remark. We can directly use the argument principle (see [10], p 1093). Indeed, the argument principle asserts the number of zeros of $L_{K}$ in $R$ is the change in argument of this function around $\partial R$ divised by $2 \pi$. Using the symmetries of $\Lambda_{K}$, we have only to consider this change on the contour $C$ (we take for example $\epsilon=1)$. The change in argument from 2 to $2+i T$ is very easy to compute because $\left|\ln \zeta_{K}(2+i t)\right| \leq N \ln \zeta(2)<N / 2$ and so, if $N \leq 3,\left|\arg \zeta_{K}(2+i t)\right|<\pi / 2$ and the argument change of $\zeta_{K}$ on this segment is $\arg \zeta_{K}(2+i T)$. Of course, if $N>3$, we replace 2 by a larger integer $k$, such that $N \ln \zeta(k)<\pi / 2$. The main problem is for the segment from $2+i T$ to $\frac{1}{2}+i T$. Here, the only solution is to compute $\Lambda_{K}(s)$ at 
a large set of points on the segment, along with its first derivatives, to show that $\Lambda_{K}(s)$ cannot change its argument by as much as $\pi$ between these points.

4.3. Results about $\Phi_{K}$. We prove here a lemma which will be used in the next section.

Lemma 4.4. We have:

$$
\begin{aligned}
& \Phi_{K}(t)=\frac{t}{2} \ln \left[\left|D_{K}\right|\left(\frac{t}{2 \pi}\right)^{N}\right]-N \frac{t}{2}-r_{1} \frac{\pi}{8}+\Theta\left(\frac{3 N}{16 t}\right), \\
& \Phi_{K}^{\prime}(t)=\frac{1}{2} \ln \left[\left|D_{K}\right|\left(\frac{t}{2 \pi}\right)^{N}\right]+\Theta\left(\frac{11 N}{48 t^{2}}\right)
\end{aligned}
$$

(recall that $-f \leq \Theta(f) \leq f$ ). Moreover, there is a number $0 \leq t_{K} \leq 7$ depending only on the number field $K$, such that $\Phi_{K}$ is monotone increasing for $t \geq t_{K}$.

Proof. Set $\tilde{B}_{k}(x)=B_{k}(x-\lfloor x\rfloor)$ and apply the Euler-Maclaurin formula. We obtain

$$
\begin{aligned}
\ln \Gamma\left(\frac{1}{4}+\frac{i t}{2}\right)= & \left(\frac{i t}{2}-\frac{1}{4}\right) \ln \frac{i t}{2}-\frac{i t}{2}+\frac{1}{2} \ln 2 \pi+\frac{B_{2}\left(\frac{1}{4}\right)}{i t} \\
& -\frac{1}{2} \int_{0}^{+\infty} \frac{B_{2}\left(x-\frac{1}{4}-\left\lfloor x-\frac{1}{4}\right\rfloor\right)}{\left(x+\frac{i t}{2}\right)^{2}} d x
\end{aligned}
$$

so, if we take imaginary part, we have

$$
\begin{aligned}
\operatorname{Im} \ln \Gamma\left(\frac{1}{4}+\frac{i t}{2}\right) & =\frac{t}{2} \ln \frac{t}{2}-\frac{t}{2}-\frac{\pi}{8}+\frac{1}{48 t}-\frac{1}{2} \operatorname{Im} \int_{0}^{+\infty} \frac{\tilde{B}_{2}\left(x-\frac{1}{4}\right)}{\left(x+\frac{i t}{2}\right)^{2}} d x \\
& =\frac{t}{2} \ln \frac{t}{2}-\frac{t}{2}-\frac{\pi}{8}+\frac{1}{48 t}+\frac{t}{2} \int_{0}^{+\infty} \frac{\tilde{B}_{2}\left(x-\frac{1}{4}\right) x}{\left(x^{2}+\frac{t^{2}}{4}\right)^{2}} d x
\end{aligned}
$$

and

$$
\operatorname{Im} \ln \Gamma\left(\frac{1}{2}+i t\right)=t \ln t-t+\frac{1}{24 t}+t \int_{0}^{+\infty} \frac{\tilde{B}_{2}\left(x-\frac{1}{2}\right) x}{\left(x^{2}+t^{2}\right)^{2}} d x
$$

and we replace in the definition of $\Phi_{K}(t)$ :

$$
\begin{aligned}
\Phi_{K}(t) & =t \ln C_{K}+\operatorname{Im}\left(r_{1} \ln \Gamma\left(\frac{1}{4}+\frac{i t}{2}\right)+r_{2} \ln \Gamma\left(\frac{1}{2}+i t\right)\right) \\
& =\frac{t}{2} \ln \left[\left|D_{K}\right|\left(\frac{t}{2 \pi}\right)^{N}\right]-N \frac{t}{2}-r_{1} \frac{\pi}{8}+\frac{N}{48 t}+J \quad(*)
\end{aligned}
$$

where

$$
|J| \leq t\left[\frac{r_{1}}{2} \int_{0}^{+\infty} \frac{\left|\tilde{B}_{2}\left(x-\frac{1}{4}\right)\right| x}{\left(x^{2}+\frac{t^{2}}{4}\right)^{2}} d x+r_{2} \int_{0}^{+\infty} \frac{\left|\tilde{B}_{2}\left(x-\frac{1}{2}\right)\right| x}{\left(x^{2}+t^{2}\right)^{2}} d x\right]
$$

and $\left|B_{2}(x)\right|=\left|x^{2}-x+\frac{1}{6}\right| \leq \frac{1}{6}$ if $x \in[0,1]$ implies that $\left|\tilde{B}_{2}(x)\right| \leq \frac{1}{6}$ if $\left.x \in\right]-\infty,+\infty[$, so

$$
\begin{aligned}
|J| & \leq \frac{t}{6}\left[\frac{r_{1}}{2} \int_{0}^{+\infty} \frac{x}{\left(x^{2}+\frac{t^{2}}{4}\right)^{2}} d x+r_{2} \int_{0}^{+\infty} \frac{x}{\left(x^{2}+t^{2}\right)^{2}} d x\right] \\
& \leq \frac{t}{6}\left[\frac{r_{1}}{2} \int_{\frac{t}{2}}^{+\infty} \frac{d u}{u^{3}}+r_{2} \int_{t}^{+\infty} \frac{d u}{u^{3}}\right] \leq \frac{r_{1}}{6 t}+\frac{r_{2}}{12 t} \leq \frac{N}{6 t}
\end{aligned}
$$

and formula (33) follows. 
To obtain the estimate for $\Phi_{K}^{\prime}$, we differentiate under the integral sign in $\left(^{*}\right)$ (see justifications in [12], p. 312)

$$
\Phi_{K}^{\prime}(t)=\frac{1}{2} \ln \left[\left|D_{K}\right|\left(\frac{t}{2 \pi}\right)^{N}\right]-\frac{N}{48 t^{2}}+J_{1}
$$

where

$$
\begin{aligned}
J_{1} & =-\frac{r_{1}}{2} \operatorname{Im} \int_{o}^{+\infty} \frac{d}{d t}\left[\frac{\tilde{B}_{2}\left(x-\frac{1}{4}\right)}{\left(x+\frac{i t}{2}\right)^{2}}\right] d x-\frac{r_{2}}{2} \operatorname{Im} \int_{0}^{+\infty} \frac{d}{d t}\left[\frac{\tilde{B}_{2}\left(x-\frac{1}{2}\right)}{(x+i t)^{2}}\right] d x \\
& =\frac{r_{1}}{2} \operatorname{Re} \int_{o}^{+\infty} \frac{\tilde{B}_{2}\left(x-\frac{1}{4}\right)\left(x-\frac{i t}{2}\right)^{3}}{\left(x^{2}+\frac{t^{2}}{4}\right)^{3}} d x+r_{2} \operatorname{Re} \int_{0}^{+\infty} \frac{B_{2}\left(x-\frac{1}{2}\right)(x-i t)^{3}}{\left(x^{2}+t^{2}\right)^{3}} d x \\
& =\frac{r_{1}}{2} \int_{0}^{+\infty} \frac{\tilde{B}_{2}\left(x-\frac{1}{4}\right)\left(x^{3}-3 x \frac{t^{2}}{4}\right)}{\left(x^{2}+\frac{t^{2}}{4}\right)^{3}} d x+r_{2} \int_{0}^{+\infty} \frac{B_{2}\left(x-\frac{1}{2}\right)\left(x^{3}-3 x t^{2}\right)}{\left(x^{2}+t^{2}\right)^{3}} d x
\end{aligned}
$$

So

$$
\left|J_{1}\right| \leq \frac{r_{1}}{24} \int_{\frac{t^{2}}{4}}^{+\infty} \frac{\left|u-t^{2}\right|}{u^{3}} d u+\frac{r_{2}}{12} \int_{t^{2}}^{+\infty} \frac{\left|u-4 t^{2}\right|}{u^{3}} d u \leq \frac{5 N}{24 t^{2}}
$$

and formula (34) follows.

We have also to verify that $\Phi_{K}^{\prime \prime}(t)>0$ for $t \geq 0$. We do not give details of the proof since it is exactly the same as in [12], Lemma 11. The idea is to write $\Phi_{K}(t)$ in the form

$$
\begin{aligned}
\Phi_{K}(t)= & -\frac{i r_{1}}{2}\left[\ln \Gamma\left(\frac{1}{4}+\frac{i t}{2}\right)-\ln \Gamma\left(\frac{1}{4}-\frac{i t}{2}\right)\right] \\
& -\frac{i r_{2}}{2}\left[\ln \Gamma\left(\frac{1}{2}+i t\right)-\ln \Gamma\left(\frac{1}{2}-i t\right)\right]+t \ln C_{K}
\end{aligned}
$$

and to differentiate twice. We then use an expansion of the $\psi$ function to show that $\Phi_{K}^{\prime \prime}(t)>0$ and thus that $\Phi_{K}^{\prime}$ is strictly increasing. We now want to prove that $\Phi_{K}^{\prime}(7)>0$. By $(34)$, it suffices to show that:

$$
\begin{aligned}
& \frac{1}{2} \ln \left[\left|D_{K}\right|\left(\frac{7}{2 \pi}\right)^{N}\right]-\frac{11 N}{48 \times 7^{2}}>0 \\
& \Leftrightarrow \quad \ln \left[\left|D_{K}\right|^{\frac{1}{N}} \frac{7}{2 \pi}\right]>\frac{11}{24 \times 7^{2}} \\
& \Leftrightarrow \quad\left|D_{K}\right|^{\frac{1}{N}}>0.906
\end{aligned}
$$

which is true for any number field. So, $\Phi_{K}$ is monotone increasing on $\left[t_{K},+\infty[\right.$ with $t_{K} \leq 7$.

4.4. Gram blocks, Gram's law and the number of zeros. We start this section by giving some definitions (for more details, see [12] , pp. 314-318). Since $\Phi_{K}$ is strictly increasing on the interval $\left[t_{K},+\infty\right.$ [, we can define the Gram points as follows. The $n^{t h}$ Gram point is the unique solution $g_{n}$ in $\left[t_{K},+\infty[\right.$ of

$$
\Phi_{K}\left(g_{n}\right)=n \pi \quad \text { for } n \geq 0
$$

and the intervals $\left.\left.G_{n}=\right] g_{n}, g_{n+1}\right]$ are called the Gram intervals. The statement that the $(n+2)^{n d}$ zero of $Z_{K}(t)$ is a real positive number in the Gram interval $G_{n}$ 
and that $Z_{K}(t)$ has no other zero in this interval is known as Gram's law. The interesting point is its connection with the behaviour of $S_{K}(t)$. Indeed, by (32) we have

$$
S_{K}\left(g_{n}\right)=N_{K}\left(g_{n}\right)-(n+1)
$$

and thus $S_{K}(t)$ takes integral values at the Gram points. Moreover, since $\Phi_{K}$ is monotone increasing, we easily see that $S_{K}$ is monotone decreasing on $\left[t_{K},+\infty\right.$, except at points $t$ which are ordinates of zeros and where $S_{K}$ jumps and increases by an integral amount (the number of zeros at height $t$ ). With these results, we can prove that Gram's law holds for a given integer $n$ if and only if $\left|S_{K}(t)\right|<1$ for $g_{n} \leq t \leq g_{n+1}$. This law is often verified but we can introduce a modified version of it which holds more frequently. A Gram block of length $l$ (see [19] for the introduction of the concept) is an interval $\left.] g_{n}, g_{n+l}\right]$ such that $(-1)^{j} Z_{K}\left(g_{j}\right)>0$ for $j=n$ and $j=n+l$, but $(-1)^{j} Z_{K}\left(g_{j}\right)<0$ for $n<j<n+l$. We say that this Gram block satisfies Rosser's rule if it contains at least $l$ zeros of $Z_{K}$. The main result of this section is based on Theorem 4.1 and on the following lemma.

Lemma 4.4. Assume that $\zeta_{K}\left(\frac{1}{2}\right)<0$ and that $t \geq 0$. Then $N_{K}(t)$ is even if $Z_{K}(t)<0$ and odd if $Z_{K}(t)>0$.

Proof. There is a slight difference with the proof of Lemma 13, in [12], p. 314, when we want to show that $Z_{K}(0)<0$. Indeed, we have $Z_{K}(0)=\zeta_{K}\left(\frac{1}{2}\right) \exp \left[i \Phi_{K}(0)\right]=$ $\zeta_{K}\left(\frac{1}{2}\right)$ (and not $\zeta_{K}(0)$ as said in [12]). The problem is to prove that $\zeta_{K}\left(\frac{1}{2}\right)<0$, and the only solution we have for this is a numerical verification with our program.

We now give the main result of this section. We start by introducing the concept of $\epsilon$-approximate Gram block. If $0 \leq \epsilon<\frac{1}{2}$ and $\left|g_{j}-j \pi\right| \leq \epsilon$ for $n \leq j \leq n+l$, then we call the interval $\left.] g_{n}, g_{n+l}\right]$ an $\epsilon$-approximate Gram block of length $l$ if $(-1)^{j} Z_{K}\left(g_{j}\right)>0$ for $j=n$ and $j=n+l$, but $(-1)^{j} Z_{K}\left(g_{j}\right)<0$ for $n<j<n+l$.

Theorem 4.4. Assume that $g_{n}>40$ and assume also that the interval $\left.] g_{n}, g_{n+l}\right]$ is the union of $p$ disjoint $\epsilon$-approximate Gram blocks, each containing at least as many zeros of $Z_{K}(t)$ as its length. If

$$
\begin{aligned}
p+\left(\frac{1}{2}-\epsilon\right) l> & (0.2928 N+0.0419) \ln \left[\left|D_{K}\right|\left(\frac{g_{n+l}}{2 \pi}\right)^{N}\right] \\
& +0.0195 \ln ^{2}\left[\left|D_{K}\right|\left(\frac{g_{n+l}}{2 \pi}\right)^{N}\right],
\end{aligned}
$$

then $N_{K}\left(g_{n}\right) \leq n+1$ and $N_{K}\left(g_{n+l}\right) \geq n+l+1$.

Proof. The result follows from Lemma 4.4 and from the following lemma (see [12], Theorem 4, pp. 317-318 for details).

Lemma 4.5. If $b>a>40$, then

$$
\begin{aligned}
\left|\int_{a}^{b} S_{K}(t) \frac{\Phi_{K}^{\prime}(t)}{\pi} d t\right| \leq & (0.2928 N+0.0419) \ln \left[\left|D_{K}\right|\left(\frac{b}{2 \pi}\right)^{N}\right] \\
& +0.0195 \ln ^{2}\left[\left|D_{K}\right|\left(\frac{b}{2 \pi}\right)^{N}\right] .
\end{aligned}
$$


Proof. We integrate by parts. We have $b>a>t_{K}\left(t_{K} \leq 7\right)$ and therefore:

$$
\begin{aligned}
\int_{a}^{b} S_{K}(t) \Phi_{K}^{\prime}(t) d t & =\Phi_{K}^{\prime}(a)\left[S_{K}^{1}(b)-S_{K}^{1}(a)\right]+\int_{a}^{b}\left[S_{K}^{1}(b)-S_{K}^{1}(t)\right] \Phi_{K}^{\prime \prime}(t) d t \\
& =\Theta\left[\max _{[a, b]}\left|S_{K}^{1}(b)-S_{K}^{1}(t)\right|\left(\left|\Phi_{K}^{\prime}(a)\right|+\int_{a}^{b}\left|\Phi_{K}^{\prime \prime}(t)\right| d t\right)\right] \\
& =\Theta\left[\max _{[a, b]}\left|S_{K}^{1}(b)-S_{K}^{1}(t)\right|\left(\frac{1}{2} \ln \left[\left|D_{K}\right|\left(\frac{b}{2 \pi}\right)^{N}\right]+\frac{11 N}{48 b^{2}}\right)\right]
\end{aligned}
$$

since $\Phi_{K}^{\prime \prime}(t)>0$ and $\Phi_{K}^{\prime}(a)>0$ when $a \geq t_{K}$. We now use Theorem 1 and we easily obtain the result claimed in Lemma 4.5. The end of the proof of Theorem 4.4 is now the same as in [12].

\section{NumERICAL COMPUTATIONS}

5.1. The method and its limitations. The first (and most expensive) part of the work is finding the zeros of $\zeta_{K}(s)$ in the interval $\left[\frac{1}{2}, \frac{1}{2}+i T\right]$. Since $Z_{K}(t)$ takes real values when $t$ is real, we only search for changes of sign of this function. We first compute $Z_{K}\left(\frac{n}{20}\right)$ for $n=0$ to $n=20 T$, we store each change of sign in a file, and then we compute the roots to accuracy $10^{-20}$.

In the second part, we rigorously prove that $Z_{K}(t)$ changes of sign at each point we have found in the first part. To do that, we use the same method as Rumely: we choose validation points between the zeros, and we re-evaluate $Z_{K}(t)$ at these points with a program which bounds roundoff error, in order to prove that $Z_{K}(t)$ really changes of sign (see 5.2 for computational details).

The third and last part consists in checking the GRH. We start by computing the second term of inequality (37) to estimate the number of Gram blocks that we need. We then compute the approximate Gram points such that $\left|g_{j}-j \pi\right| \leq 0.1$ and we verify that each Gram block contains the correct number of zeros. We finally prove the GRH using Theorem 4.4.

In the remainder of this section, we point out the limitations of our method that led us to choose $T=92$ for cubic fields and $T=40$ for quartic fields.

The first limitation is due to formulas (5) and (6) (or formula (16) for the second form of the method) since the number of significant digits is limited by the largest element occurring in the course of a summation. Indeed, the maximal term in these sums is very large compared to the final answer, so we have to increase the internal precision in our computation. If we want to compute $\Lambda_{K}(s)$ with an absolute precision $\epsilon$, we have to compute $f\left(\frac{C_{K}}{n}, s\right)$ for $n=1$ to $N_{0}$ such that $\left.\mid f\left(\frac{C_{K}}{N_{0}}, s\right)\right) \mid<\frac{\epsilon}{2 M}$ (see Section 3.2). We search for the largest element that occurs in formula (5) when $x=x_{0}=\frac{C_{K}}{N_{0}}$. In the following $f_{k}\left(x_{0}, s\right)$ denotes the sum in (5) truncated to $k$ terms. From the proof of Proposition 3.3 we have

$$
\begin{aligned}
\left|f_{k}\left(x_{0}, s\right)\right| & \leq \frac{\left(\frac{N_{0}}{C_{K}}\right)^{k+\alpha}(2 \sqrt{\pi})^{r_{2}} 5^{r_{1}}}{\Gamma\left(\frac{k}{2}+1\right)^{r_{1}} \Gamma(k+1)^{r_{2}} \pi^{\frac{3}{2}}} \quad \text { with } 0<\alpha \leq \frac{1}{2} \\
& \leq\left(\frac{N_{0}}{C_{K}}\right)^{\alpha} \frac{(2 \sqrt{\pi})^{r_{2}} 5^{r_{1}}}{\pi^{\frac{3}{2}} \sqrt{2 \pi}^{r_{1}+r_{2}}} \exp \left[k\left(\ln \frac{N_{0}}{C_{K}}+\frac{N}{2}+\frac{r_{1}}{2} \ln 2\right)-\frac{N}{2} k \ln k\right] .
\end{aligned}
$$


We introduce the function

$$
g(k)=\exp \left[k\left(\ln \frac{N_{0}}{C_{K}}+\frac{N}{2}+\frac{r_{1}}{2} \ln 2\right)-\frac{N}{2} k \ln k\right]
$$

and we easily see that

$$
g^{\prime}(k)=0 \quad \Leftrightarrow \quad k=\left(\frac{N_{0}}{C_{K}}\right)^{\frac{2}{N}} 2^{\frac{r_{1}}{N}}
$$

and that

$$
\left|f_{k}(x, s)\right| \leq\left(\frac{N_{0}}{C_{K}}\right)^{\alpha} \frac{(2 \sqrt{\pi})^{r_{2}} 5^{r_{1}}}{\pi^{\frac{3}{2}} \sqrt{2 \pi}^{r_{1}+r_{2}}} \exp \left[\frac{N}{2}\left(\frac{N_{0}}{C_{K}}\right)^{\frac{2}{N}} 2^{\frac{r_{1}}{N}}\right] \quad \forall 0<\alpha<\frac{1}{2}
$$

and using the relation (17), we obtain after some computations that

$$
\left|f_{k}(x, s)\right| \leq \frac{2 M 5^{N}}{\epsilon} \quad \text { for all } k \leq i_{0} \quad \text { and } \quad \frac{C_{K}}{N_{0}} \leq x \leq C_{K}
$$

where $M$ is a bound for the number of ideals. We use this result as follows: if we want a value of $\Lambda_{K}(s)$ with $k$ significant digits $\left(\epsilon=10^{-k}\right)$, we make the computations with $2 k+c$ digits (with $c \ln 10=\ln 2 M 5^{N}$ ) to be sure that the result is correct.

The second limitation is due to the gamma factors in (2). The absolute value of $\Lambda_{K}\left(\frac{1}{2}+i t\right)$ decreases exponentially with $t$. Indeed, the modulus of $\Lambda_{K}\left(\frac{1}{2}+i t\right)$ is essentially the modulus of its gamma factors. Using Stirling's formula, we easily obtain

$$
\left|\Lambda_{K}\left(\frac{1}{2}+i t\right)\right|=\mathcal{O}\left(\exp \left[-\frac{N \pi t}{4}\right]\right) .
$$

For example, if we work with a number field of degree 3 , and if we set $T=100$, we have $\exp (-300 \pi / 4) \leq 5.10^{-103}$ so if we want to compute a root to accuracy $10^{-20}$, we must work with $103+20=123$ digits, and we must increase this accuracy to more than 200 digits (see preceding paragraph).

The last limitation is in the degree (and the discriminant) of the number field. Indeed, the value of $N_{0}$ increases with $N$ or $D_{K}$. For large values of $N_{0}$, the most expensive part of the program is the computation of the sum $\sum_{n=1}^{N_{0}} a_{n} n^{-s}$ in formula (14), and it seems that there is no solution to improve this computation.

5.2. The roundoff error. To estimate the accumulated roundoff error, we follow the same method as Rumely ([20] pp. 423-425). We use another program which re-evaluates $Z_{K}(t)$ at validation points (see 5.1) and which computes the roundoff error in the same time. In this program, we represent a real number $\alpha$ by a pair $\left(a, \epsilon_{a}\right)$ which means that $a-\epsilon_{a} \leq \alpha \leq a+\epsilon_{a}$ and we determine how the error term propagates through the computations (we consider a complex number as a pair $\left.\left(\left(x, \epsilon_{x}\right),\left(y, \epsilon_{y}\right)\right)\right)$. We use for this the formulas given in [20] p. 425 for the principal operations (addition, multiplication, exponentiation...). For example, we apply this method to the first term in formula (14). 
If $A(s)=\frac{2_{1}^{r} h_{K} R_{K}}{\omega_{K}(s-1) s}$ and $h_{K}, R_{K}, s=\frac{1}{2}+i t$ are given with a relative precision $\epsilon_{0}=10^{-k}$, we have

$$
\epsilon_{A} \leq\left[\frac{2^{r_{1}+3} h_{K} R_{K}}{\omega_{K}} \times \frac{t^{2}+1}{t^{2}-\frac{1}{4}}\right] \epsilon_{0}+\left[\frac{2^{r_{1}+2} h_{K} R_{K}}{\omega_{K}} \times \frac{t^{2}}{t^{2}-\frac{1}{4}}+\frac{2^{r_{1}+1} h_{K} R_{K}}{\omega_{K}}+2\right] \epsilon_{0}^{2} .
$$

Our program uses this style of formulas and combines them with numerical computations to obtain a bound of roundoff error of each term in formula (14). We do not give here all the computational details since it is rather long and has little interest, but we have obtained the following results for cubic fields.

Suppose that we want to obtain values of $\zeta_{K}(s)(\operatorname{Im} s \leq 100)$ with 20 digits. We know that $\zeta_{K}\left(\frac{1}{2}+100 i\right) \simeq 10^{-100}$ and so, we set $\epsilon=10^{-120}$ to compute $N_{0}$ and $i_{0}$. We suppose now that $h_{K}, R_{K}, s$ and $\pi$ are given with a relative precision $\epsilon_{0}$ and we have

- $A=\frac{2^{r_{1}} h_{K} R_{K}}{\omega_{K}(s-1) s} \quad \epsilon_{A} \leq 635 \epsilon_{0}$.

- $B=C_{K}^{s} \Gamma\left(\frac{s}{2}\right)^{r_{1}} \Gamma(s)^{r_{2}} \sum_{n>1} a_{n} n^{-s} \quad \epsilon_{B} \leq 6.10^{7} \epsilon_{0}$.

(We also have $\epsilon_{C} \leq 6.10^{7} \epsilon_{0}$ if $C=C_{K}^{1-s} \Gamma\left(\frac{1-s}{2}\right)^{r_{1}} \Gamma(1-s)^{r_{2}} \sum_{n \geq 1} a_{n} n^{s-1}$.)

- $D=\sum_{k=0}^{r_{1}+r_{2}-1} \sum_{i=0}^{i_{0}} \frac{c_{i, k}}{(s+i)^{k+1}} \quad \epsilon_{D} \leq 4.10^{128} \epsilon_{0}$.

(We also have $\epsilon_{E} \leq 4.10^{128} \epsilon_{0}$ if $E=\sum_{k=0}^{r_{1}+r_{2}-1} \sum_{i=0}^{i_{0}} \frac{c_{i, k}}{(1-s+i)^{k+1}}$.)

From this, we can deduce that

$$
\left|\tilde{\zeta}_{K}(s)-\zeta_{K}(s)\right| \leq 5.10^{128} \epsilon_{0}
$$

where $\tilde{\zeta}_{K}(s)$ is the result of our computation, and $\zeta_{K}(s)$ is the exact value. So, to be sure that we really have a zero between two validation points, we need that $5.10^{128} \epsilon_{0}=10^{-120}$, and thus, we compute $\zeta_{K}(s)$ at these points with 249 digits $\left(\epsilon=10^{-120}\right.$ and $\left.\epsilon_{0}=10^{-249}\right)$.

Numerical results are given in section 6 (50 cubic fields, 30 quartic fields). For each field, we have proved the GRH (height 92 for degree 3 and 40 for degree 4 ) and we give the first zero and the least and greatest gap between zeros (the complete list of zeros is available upon request from the author). The functions $\zeta_{K}(s)$ and $\Lambda_{K}(s)$ are now included in the GP/Pari calculator. These functions receive a polynomial $T$, a complex number $s$, and compute the value of $\zeta_{K}$ or $\Lambda_{K}$ at $s$.

\section{Numerical Results}

For number fields of degree 3 and 4 , we have proved the GRH up to a certain height. For each field, we give this height, the first zero and the least and greatest gap between two successive zeros. When the degree of the field is 5 or 6 , we only give a table of small zeros because of the limitations of our program (see Section $6.7)$. 
6.1. Degree 3 and signature $(3,0)$.

\begin{tabular}{|c|c|c|c|c|c|c|c|c|}
\hline$P(x)$ & $D_{K}$ & $\begin{array}{c}\text { GRH } \\
\text { Height }\end{array}$ & $\begin{array}{r}\mathrm{Nb} \\
\leq 92 \\
\end{array}$ & $\begin{array}{l}\text { First } \\
\text { Zero } \\
\end{array}$ & $\begin{array}{c}\text { Least } \\
\text { Gap }\end{array}$ & $\begin{array}{c}\text { Greatest } \\
\text { Gap }\end{array}$ & $\begin{array}{l}\text { Least } \\
\text { Gap* }\end{array}$ & $\begin{array}{c}\text { Greatest } \\
\text { Gap** }\end{array}$ \\
\hline$x^{3}+x^{2}-2 x-1$ & 49 & 94.31 & 131 & 4.35640 & 0.00270 & 1.95056 & 0.00270 & 2.10758 \\
\hline$x^{3}-3 x-1$ & 81 & 93.54 & 139 & 3.44409 & 0.02293 & 2.21285 & 0.02519 & 2.21285 \\
\hline$x^{3}+x^{2}-3 x-1$ & 148 & 94.03 & 147 & 3.02402 & 0.00435 & 1.85461 & 0.09947 & 1.85461 \\
\hline$x^{3}-x^{2}-4 x-1$ & 169 & 92.13 & 149 & 2.27313 & 0.00545 & 2.66545 & 0.00545 & 2.66545 \\
\hline$x^{3}-4 x-1$ & 229 & 93.78 & 154 & 2.61412 & 0.01298 & 1.75660 & 0.05787 & 1.75660 \\
\hline$x^{3}-5 x-3$ & 257 & 93.91 & 155 & 2.12932 & 0.00273 & 2.05906 & 0.05829 & 2.05906 \\
\hline$x^{3}+x^{2}-4 x-2$ & 316 & 93.42 & 158 & 2.86162 & 0.02098 & 1.64006 & 0.15851 & 1.64006 \\
\hline$x^{3}+x^{2}-4 x-1$ & 321 & 93.31 & 159 & 2.21077 & 0.01432 & 2.17808 & 0.06883 & 2.17808 \\
\hline$x^{3}+x^{2}-6 x-7$ & 361 & 93.87 & 161 & 1.57320 & 0.00429 & 2.83562 & 0.02451 & 2.83562 \\
\hline$x^{3}-x^{2}-5 x-1$ & 404 & 93.57 & 162 & 2.28061 & 0.03555 & 1.72736 & 0.06728 & 1.72736 \\
\hline$x^{3}+x^{2}-5 x-4$ & 469 & 94.35 & 164 & 2.05522 & 0.00920 & 1.63356 & 0.07146 & 1.63356 \\
\hline$x^{3}-5 x-1$ & 473 & 92.98 & 164 & 1.72932 & 0.00453 & 1.93468 & 0.12857 & 1.93468 \\
\hline$x^{3}+x^{2}-5 x-3$ & 564 & 94.02 & 166 & 2.40567 & 0.01047 & 1.46857 & 0.17307 & 1.46857 \\
\hline$x^{3}-x^{2}-6 x-2$ & 568 & 93.54 & 167 & 2.38573 & 0.01107 & 1.40286 & 0.14241 & 1.40286 \\
\hline$x^{3}-6 x-3$ & 621 & 94.26 & 168 & 2.23358 & 0.00908 & 1.29944 & 0.16193 & 1.29944 \\
\hline$x^{3}-x^{2}-8 x-5$ & 697 & 94.38 & 170 & 1.26559 & 0.00486 & 2.11886 & 0.13953 & 2.11886 \\
\hline$x^{3}+x^{2}-7 x-8$ & 733 & 93.63 & 171 & 1.90179 & 0.00294 & 1.45064 & 0.14844 & 1.45064 \\
\hline$x^{3}-6 x-2$ & 756 & 93.86 & 171 & 1.90188 & 0.04830 & 1.52764 & 0.10620 & 1.52764 \\
\hline$x^{3}-x^{2}-6 x-1$ & 761 & 93.81 & 171 & 1.43397 & 0.02358 & 1.89358 & 0.08302 & 1.89358 \\
\hline$x^{3}+x^{2}-6 x-5$ & 785 & 93.19 & 172 & 1.63471 & 0.00814 & 1.77858 & 0.11881 & 1.77858 \\
\hline$x^{3}-x^{2}-7 x-3$ & 788 & 93.59 & 172 & 1.93396 & 0.00781 & 1.43374 & 0.09976 & 1.43374 \\
\hline$x^{3}-6 x-1$ & 837 & 94.06 & 172 & 2.16814 & 0.01414 & 1.34862 & 0.11652 & 1.34862 \\
\hline$x^{3}+x^{2}-8 x-10$ & 892 & 92.81 & 173 & 2.10560 & 0.00499 & 1.39146 & 0.09983 & 1.39146 \\
\hline$x^{3}-7 x-4$ & 940 & 93.75 & 175 & 2.20619 & 0.01679 & 1.49646 & 0.10598 & 1.49646 \\
\hline$x^{3}+x^{2}-10 x-8$ & 961 & 93.19 & 175 & 2.59082 & 0.00529 & 1.98059 & 0.00529 & 1.98059 \\
\hline
\end{tabular}

This table gives statistics for the first 25 cubic fields with signature $(3,0)$. Columns 8 and 9 give the least and greatest gap between two successive zeros, without zeros of the Riemann zeta function. For each field, we needed between 9 and 13 Gram blocks satisfying Rosser's rule, to check the GRH. We have found no exception to this rule during our computations. Table 6.2 gives exactly the same results for cubic fields of signature $(1,1)$.

In Section 6.3, we have computed the height $h_{0}\left(D_{K}\right)$ of the first zero of 1000 cubic fields of each signature and we have plotted the function

$$
f\left(D_{K}\right)=h_{0}\left(D_{K}\right) \times \ln \left|D_{K}\right|
$$

to verify that $h_{0}\left(D_{K}\right)=\mathcal{O}\left(\frac{1}{\ln \left|D_{K}\right|}\right)$.

6.2. Degree 3 and signature $(1,1)$.

\begin{tabular}{|c|c|c|c|c|c|c|c|c|}
\hline$P(x)$ & $D_{K}$ & $\begin{array}{c}\text { GRH } \\
\text { Height }\end{array}$ & $\begin{array}{r}\mathrm{Nb} \\
\leq 92 \\
\end{array}$ & $\begin{array}{l}\text { First } \\
\text { Zero }\end{array}$ & $\begin{array}{c}\text { Least } \\
\text { Gap }\end{array}$ & $\begin{array}{c}\text { Greatest } \\
\text { Gap }\end{array}$ & $\begin{array}{l}\text { Least } \\
\text { Gap* }\end{array}$ & $\begin{array}{c}\text { Greatest } \\
\text { Gap* }\end{array}$ \\
\hline$x^{3}+x^{2}-1$ & -23 & 93.81 & 121 & 5.11568 & 0.04081 & 2.04357 & 0.25629 & 2.04357 \\
\hline$x^{3}-x^{2}-1$ & -31 & 94.11 & 125 & 4.16621 & 0.00897 & 2.75950 & 0.28513 & 2.75950 \\
\hline$x^{3}-x^{2}-x-1$ & -44 & 93.98 & 130 & 4.04199 & 0.00313 & 1.77366 & 0.17532 & 1.77366 \\
\hline$x^{3}+2 x-1$ & -59 & 93.78 & 134 & 3.43180 & 0.02993 & 2.21542 & 0.09515 & 2.21542 \\
\hline$x^{3}-2 x-2$ & -76 & 93.89 & 138 & 3.27623 & 0.02791 & 1.92620 & 0.13147 & 1.92620 \\
\hline$x^{3}-x^{2}+x-2$ & -83 & 93.73 & 139 & 2.91606 & 0.04557 & 2.33787 & 0.11248 & 2.33787 \\
\hline$x^{3}+x^{2}+2 x-1$ & -87 & 93.38 & 140 & 2.68368 & 0.00008 & 2.29417 & 0.17799 & 2.29417 \\
\hline$x^{3}-x-2$ & -104 & 93.06 & 142 & 3.31991 & 0.03118 & 1.90971 & 0.10893 & 1.90971 \\
\hline$x^{3}-x^{2}+3 x-2$ & -107 & 93.34 & 143 & 2.61608 & 0.06722 & 2.30193 & 0.15955 & 2.30193 \\
\hline$x^{3}-2$ & -108 & 94.26 & 143 & 2.82161 & 0.02491 & 1.71850 & 0.19064 & 1.71850 \\
\hline$x^{3}-x^{2}-2$ & -116 & 93.73 & 144 & 3.11568 & 0.01069 & 2.11816 & 0.06693 & 2.11816 \\
\hline$x^{3}+3 x-1$ & -135 & 93.12 & 146 & 2.04028 & 0.00050 & 2.36295 & 0.08348 & 2.36295 \\
\hline$x^{3}+x^{2}+x-2$ & -139 & 93.40 & 146 & 2.66179 & 0.00171 & 1.90768 & 0.12775 & 1.90768 \\
\hline$x^{3}+2 x-2$ & -140 & 93.35 & 147 & 2.27790 & 0.02046 & 2.59247 & 0.05948 & 2.59247 \\
\hline$x^{3}-x^{2}-2 x-2$ & -152 & 94.20 & 148 & 2.81196 & 0.00546 & 1.91305 & 0.17549 & 1.91305 \\
\hline$x^{3}+x^{2}-x-3$ & -172 & 93.79 & 150 & 2.35670 & 0.00616 & 1.84139 & 0.13394 & 1.84139 \\
\hline$x^{3}-x^{2}+2 x-3$ & -175 & 93.20 & 150 & 1.81080 & 0.02071 & 2.39880 & 0.18226 & 2.39880 \\
\hline$x^{3}-x^{2}+4 x-1$ & -199 & 92.30 & 152 & 1.67161 & 0.01448 & 2.62086 & 0.19531 & 2.62086 \\
\hline$x^{3}+x^{2}+2 x-2$ & -200 & 93.67 & 152 & 2.52234 & 0.01052 & 1.78618 & 0.11447 & 1.78618 \\
\hline$x^{3}-x^{2}+x-3$ & -204 & 93.07 & 152 & 2.51256 & 0.00346 & 1.84822 & 0.08541 & 1.84822 \\
\hline$x^{3}-2 x-3$ & -211 & 92.83 & 153 & 2.29906 & 0.01656 & 1.68544 & 0.12543 & 1.68544 \\
\hline$x^{3}-x^{2}+4 x-2$ & -212 & 93.27 & 152 & 2.46540 & 0.02508 & 1.79746 & 0.10591 & 1.79746 \\
\hline$x^{3}+3 x-2$ & -216 & 94.07 & 153 & 2.79656 & 0.00312 & 1.62192 & 0.06707 & 1.62192 \\
\hline$x^{3}+x^{2}-3$ & -231 & 93.14 & 154 & 1.84481 & 0.00523 & 2.34089 & 0.12322 & 2.34089 \\
\hline$x^{3}-x-3$ & -239 & 93.83 & 154 & 2.12721 & 0.01478 & 2.69767 & 0.15969 & 2.69767 \\
\hline
\end{tabular}




\subsection{Statistics about the height of the first zero.}
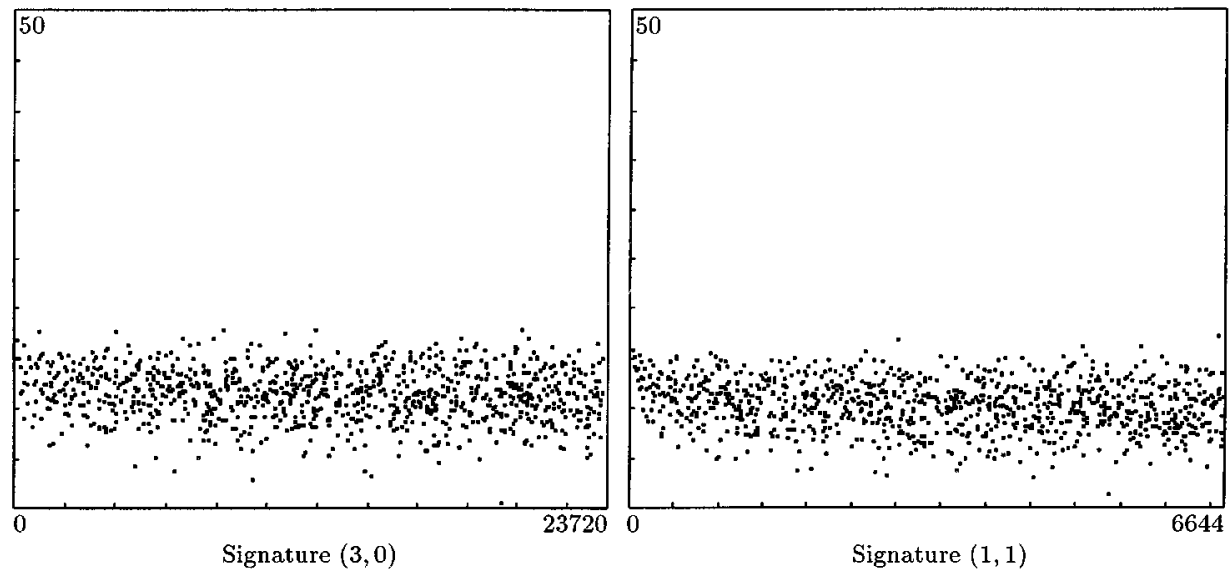

6.4. Statistics for cubic fields to height 40. This table gives statistics for cubic fields to height 40 . We give it only to compare results with tables of degree 4.

\begin{tabular}{|l|c|c|c|c|c|}
\hline$D_{K}$ & $\begin{array}{c}\text { Nb } \\
\leq 40\end{array}$ & $\begin{array}{c}\text { Least } \\
\text { Gap }\end{array}$ & $\begin{array}{c}\text { Greatest } \\
\text { Gap }\end{array}$ & $\begin{array}{c}\text { Least } \\
\text { Gap* }\end{array}$ & $\begin{array}{c}\text { Greatest } \\
\text { Gap* }\end{array}$ \\
\hline 49 & 41 & 0.00932 & 1.95056 & 0.00932 & 2.10758 \\
81 & 45 & 0.02293 & 2.21285 & 0.05483 & 2.21285 \\
148 & 49 & 0.09464 & 1.85461 & 0.25450 & 1.85461 \\
169 & 49 & 0.04901 & 2.66545 & 0.04901 & 2.66545 \\
229 & 52 & 0.03159 & 1.75660 & 0.20172 & 1.75660 \\
257 & 52 & 0.04456 & 2.05906 & 0.16923 & 2.05906 \\
316 & 53 & 0.03330 & 1.64006 & 0.23388 & 1.64006 \\
321 & 54 & 0.02999 & 2.17808 & 0.17970 & 2.17808 \\
361 & 54 & 0.02451 & 2.83562 & 0.02451 & 2.83562 \\
404 & 55 & 0.14017 & 1.72736 & 0.24106 & 1.72736 \\
469 & 56 & 0.00920 & 1.63356 & 0.21808 & 1.63356 \\
473 & 56 & 0.01432 & 1.93468 & 0.16644 & 1.93468 \\
564 & 57 & 0.04620 & 1.46857 & 0.17307 & 1.46857 \\
568 & 57 & 0.12967 & 1.40286 & 0.16699 & 1.40286 \\
621 & 58 & 0.01838 & 1.29944 & 0.16193 & 1.29944 \\
697 & 58 & 0.01390 & 2.11886 & 0.13953 & 2.11886 \\
733 & 59 & 0.00574 & 1.45064 & 0.14844 & 1.45064 \\
756 & 59 & 0.04841 & 1.52764 & 0.10620 & 1.52764 \\
761 & 59 & 0.04487 & 1.89358 & 0.16024 & 1.89358 \\
785 & 59 & 0.03064 & 1.77858 & 0.23442 & 1.77858 \\
788 & 59 & 0.04555 & 1.43374 & 0.20056 & 1.43374 \\
837 & 60 & 0.04358 & 1.34862 & 0.20740 & 1.34862 \\
892 & 60 & 0.02110 & 1.39146 & 0.20080 & 1.39146 \\
940 & 60 & 0.02855 & 1.49646 & 0.10598 & 1.49646 \\
961 & 60 & 0.00943 & 1.98059 & 0.02896 & 1.98059 \\
\hline
\end{tabular}

\begin{tabular}{|l|c|c|c|c|c|}
\hline$D_{K}$ & $\begin{array}{c}\text { Nb } \\
\leq 40\end{array}$ & $\begin{array}{c}\text { Least } \\
\text { Gap }\end{array}$ & $\begin{array}{c}\text { Greatest } \\
\text { Gap }\end{array}$ & $\begin{array}{c}\text { Least } \\
\text { Gap* }\end{array}$ & $\begin{array}{c}\text { Greatest } \\
\text { Gap* }\end{array}$ \\
\hline-23 & 37 & 0.04081 & 2.04357 & 0.43639 & 2.04357 \\
-31 & 39 & 0.06966 & 2.75950 & 0.33564 & 2.75950 \\
-44 & 41 & 0.08500 & 1.77366 & 0.17532 & 1.77366 \\
-59 & 43 & 0.08438 & 2.21542 & 0.09515 & 2.21542 \\
-76 & 45 & 0.02791 & 1.92620 & 0.13147 & 1.92620 \\
-83 & 45 & 0.07893 & 2.33787 & 0.24891 & 2.33787 \\
-87 & 45 & 0.00008 & 2.29417 & 0.27303 & 2.29417 \\
-104 & 47 & 0.16163 & 1.90971 & 0.16163 & 1.90971 \\
-107 & 47 & 0.07351 & 2.30193 & 0.27553 & 2.30193 \\
-108 & 47 & 0.02491 & 1.71850 & 0.23129 & 1.71850 \\
-116 & 47 & 0.09699 & 2.11816 & 0.22768 & 2.11816 \\
-135 & 48 & 0.05242 & 2.36295 & 0.08348 & 2.36295 \\
-139 & 49 & 0.10583 & 1.90768 & 0.12775 & 1.90768 \\
-140 & 48 & 0.08975 & 2.59247 & 0.29068 & 2.59247 \\
-152 & 49 & 0.04671 & 1.91305 & 0.25602 & 1.91305 \\
-172 & 49 & 0.11080 & 1.84139 & 0.13394 & 1.84139 \\
-175 & 50 & 0.02236 & 2.39880 & 0.27872 & 2.39880 \\
-199 & 50 & 0.01448 & 2.62086 & 0.19531 & 2.62086 \\
-200 & 51 & 0.01052 & 1.78618 & 0.11447 & 1.78618 \\
-204 & 51 & 0.00522 & 1.84822 & 0.24845 & 1.84822 \\
-211 & 51 & 0.01656 & 1.68544 & 0.13736 & 1.68544 \\
-212 & 51 & 0.09019 & 1.79746 & 0.14141 & 1.79746 \\
-216 & 51 & 0.05687 & 1.62192 & 0.06707 & 1.62192 \\
-231 & 51 & 0.03945 & 2.34089 & 0.32738 & 2.34089 \\
-239 & 52 & 0.01913 & 2.69767 & 0.16827 & 2.69767 \\
\hline
\end{tabular}

6.5. Degree 4 and signature $(4,0)$.

\begin{tabular}{|l|c|c|c|c|c|c|c|c|}
\hline \multicolumn{1}{|c|}{$P(x)$} & $D_{K}$ & $\begin{array}{c}\text { GRH } \\
\text { Height }\end{array}$ & $\begin{array}{c}\text { Nb } \\
\leq 40\end{array}$ & $\begin{array}{c}\text { First } \\
\text { Zero }\end{array}$ & $\begin{array}{c}\text { Least } \\
\text { Gap }\end{array}$ & $\begin{array}{c}\text { Greatest } \\
\text { Gap }\end{array}$ & $\begin{array}{c}\text { Least } \\
\text { Gap* }\end{array}$ & $\begin{array}{c}\text { Greatest } \\
\text { Gap* }\end{array}$ \\
\hline$x^{4}+x^{3}-3 x^{2}-x+1$ & 725 & 42.62 & 65 & 3.31527 & 0.01457 & 1.25258 & 0.29755 & 1.69636 \\
$x^{4}+x^{3}-4 x^{2}-4 x+1$ & 1125 & 40.91 & 67 & 2.73460 & 0.00729 & 1.67209 & 0.11973 & 1.77291 \\
$x^{4}+2 x^{3}-7 x^{2}+2 x+1$ & 1600 & 42.05 & 70 & 2.48821 & 0.00483 & 1.54338 & --- & --- \\
$x^{4}-4 x^{2}+x+1$ & 1957 & 42.73 & 70 & 2.52928 & 0.01406 & 1.24419 & 0.10595 & 1.24419 \\
$x^{4}-5 x^{2}+5$ & 2000 & 40.60 & 71 & 2.43970 & 0.00459 & 1.83982 & 0.00721 & 1.83982 \\
$x^{4}-4 x^{2}+2$ & 2048 & 40.95 & 71 & 2.84244 & 0.00315 & 1.35797 & 0.00315 & 1.97953 \\
$x^{4}+x^{3}-5 x^{2}-2 x+4$ & 2225 & 42.37 & 72 & 2.49859 & 0.00718 & 1.62549 & 0.25728 & 1.62549 \\
$x^{4}-4 x^{2}+1$ & 2304 & 41.46 & 72 & 2.68865 & 0.00993 & 1.39979 & --- & --- \\
$x^{4}+x^{3}-6 x^{2}+5$ & 2525 & 41.62 & 72 & 2.13772 & 0.01125 & 1.45974 & 0.22223 & 1.55324 \\
$x^{4}+2 x^{3}-3 x^{2}-2 x+1$ & 2624 & 41.51 & 72 & 2.15522 & 0.00149 & 1.38864 & 0.13669 & 1.88352 \\
\hline
\end{tabular}


6.6. Degree 4 and signature $(2,1)$.

\begin{tabular}{|l|c|c|c|c|c|c|c|c|}
\hline \multicolumn{1}{|c|}{$P(x)$} & $D_{K}$ & $\begin{array}{c}\text { GRH } \\
\text { Height }\end{array}$ & $\begin{array}{c}\text { Nb } \\
\leq 40\end{array}$ & $\begin{array}{c}\text { First } \\
\text { Zero }\end{array}$ & $\begin{array}{c}\text { Least } \\
\text { Gap }\end{array}$ & $\begin{array}{c}\text { Greatest } \\
\text { Gap }\end{array}$ & $\begin{array}{c}\text { Least } \\
\text { Gap* }\end{array}$ & $\begin{array}{c}\text { Greatest } \\
\text { Gap** }\end{array}$ \\
\hline$x^{4}+x^{3}-2 x-1$ & -275 & 41.35 & 58 & 3.81577 & 0.00649 & 1.54002 & 0.14005 & 2.19745 \\
$x^{4}+x-1$ & -283 & 43.62 & 58 & 3.72299 & 0.00527 & 1.52318 & 0.21046 & 1.52318 \\
$x^{4}+x^{3}-x^{2}-x-1$ & -331 & 43.12 & 59 & 3.40271 & 0.01425 & 1.91807 & 0.17352 & 1.91807 \\
$x^{4}-x^{2}-1$ & -400 & 42.06 & 61 & 3.18874 & 0.03688 & 1.65784 & 0.20874 & 1.65784 \\
$x^{4}+2 x^{3}+x^{2}-2 x-1$ & -448 & 42.17 & 62 & 3.00942 & 0.01153 & 1.89054 & 0.26119 & 2.84525 \\
$x^{4}+x^{3}-2 x^{2}+2 x-1$ & -475 & 42.45 & 62 & 2.87350 & 0.00875 & 2.18414 & 0.29969 & 2.18414 \\
$x^{4}+x^{3}-2 x^{2}-2 x-1$ & -491 & 43.25 & 62 & 3.18155 & 0.11444 & 1.56191 & 0.22678 & 1.56191 \\
$x^{4}+x^{3}-x^{2}+x+1$ & -507 & 42.25 & 62 & 3.11934 & 0.01349 & 1.50662 & 0.38555 & 1.81562 \\
$x^{4}+x^{3}-x^{2}+x-1$ & -563 & 42.83 & 63 & 2.96180 & 0.05622 & 1.46111 & 0.16249 & 1.46111 \\
$x^{4}+x^{3}+2 x+1$ & -643 & 42.43 & 63 & 2.53414 & 0.00415 & 2.19025 & 0.17572 & 2.19025 \\
\hline
\end{tabular}

6.7. Degree 4 and signature $(0,2)$.

\begin{tabular}{|l|c|c|c|c|c|c|c|c|}
\hline \multicolumn{1}{|c|}{$P(x)$} & $D_{K}$ & $\begin{array}{c}\text { GRH } \\
\text { Height }\end{array}$ & $\begin{array}{c}\text { Nb } \\
\leq 40\end{array}$ & $\begin{array}{c}\text { First } \\
\text { Zero }\end{array}$ & $\begin{array}{c}\text { Least } \\
\text { Gap }\end{array}$ & $\begin{array}{c}\text { Greatest } \\
\text { Gap }\end{array}$ & $\begin{array}{c}\text { Least } \\
\text { Gap* }\end{array}$ & $\begin{array}{c}\text { Greatest } \\
\text { Gap* }\end{array}$ \\
\hline$x^{4}+x^{3}-x^{2}-x+1$ & 117 & 42.05 & 53 & 4.39582 & 0.00758 & 1.81562 & 0.38555 & 1.81562 \\
$x^{4}+x^{3}+x^{2}+x+1$ & 125 & 41.82 & 53 & 4.13290 & 0.00019 & 2.05067 & 0.00019 & 2.27365 \\
$x^{4}-x^{2}+1$ & 144 & 41.85 & 54 & 3.80462 & 0.02192 & 2.21632 & --- & -- \\
$x^{4}+x^{3}-2 x+1$ & 189 & 42.92 & 56 & 3.51328 & 0.03331 & 1.64927 & 0.27429 & 1.71614 \\
$x^{4}+x^{3}+2 x^{2}-x+1$ & 225 & 41.87 & 58 & 3.05701 & 0.00200 & 2.28617 & --- & -- \\
$x^{4}+x+1$ & 229 & 42.77 & 57 & 3.19363 & 0.03188 & 1.85968 & 0.14911 & 1.85968 \\
$x^{4}+1$ & 256 & 41.45 & 58 & 3.57615 & 0.00590 & 1.87477 & --- & --- \\
$x^{4}+x^{2}+x+1$ & 257 & 41.92 & 58 & 3.56819 & 0.01113 & 1.34834 & 0.17364 & 1.34834 \\
$x^{4}+x^{2}+2 x+1$ & 272 & 41.74 & 58 & 2.75562 & 0.01938 & 2.65702 & 0.25713 & 2.65702 \\
$x^{4}+2 x^{3}+2$ & 320 & 41.23 & 59 & 3.18874 & 0.01303 & 1.65282 & 0.20874 & 1.65784 \\
\hline
\end{tabular}

We give for quartic fields exactly the same results as in 6.1 and 6.2 but we have stopped our computations at height 40 (see Section 5). Columns 8 and 9 give the least and greatest gap between two successive zeros without all zeros of the Riemann zeta function, and without all zeros of Dedekind zeta functions of subfields. If there is no value, this means that each zero of the Dedekind zeta function is also a zero of the Dedekind zeta function of a subfield.

\subsection{Low zeros in degree 5 and degree 6 .}

\begin{tabular}{|c|c|c|c|c|c|c|}
\hline$(5,0)$ & $(3,1)$ & $(1,2)$ & $(6,0)$ & $(4,1)$ & $(2,2)$ & $(0,3)$ \\
14641 & -4511 & 1609 & 300125 & -92779 & 28037 & -9747 \\
\hline 2.6960041 & 3.0959276 & 3.5046434 & 2.6107359 & 2.6567860 & 3.0348978 & 3.3084337 \\
3.6100404 & 3.9839167 & 4.4029081 & 2.8054153 & 3.2834471 & 3.4842283 & 3.8933927 \\
4.6293537 & 5.1824400 & 5.8558274 & 4.0919419 & 4.2570181 & 4.9815596 & 5.4260008 \\
5.1336996 & 5.8433821 & 6.3798009 & 4.3564016 & 4.8393463 & 5.1156833 & 5.5084686 \\
6.0318093 & 6.5240485 & 7.1361800 & 4.9665914 & 5.4475842 & 5.8117296 & 6.5621326 \\
6.7062198 & 7.4281911 & 8.2402966 & 5.5951657 & 6.0325920 & 6.8129660 & 7.0204644 \\
7.2069265 & 7.9450296 & 8.7206690 & 6.2012300 & 6.5817773 & 7.1592623 & 8.0397372 \\
7.6618576 & 8.6508744 & 9.3318488 & 6.6484533 & 7.0862482 & 7.5677683 & 8.1130254 \\
8.7041611 & 9.2160990 & & 6.8272084 & 7.7047603 & 8.3161065 & 8.9799068 \\
9.0057129 & 9.8659138 & & 7.7709912 & 8.1083577 & 8.8313966 & 9.5307146 \\
9.3257628 & & & 7.9274309 & 8.4529841 & 9.1792851 & 9.7559341 \\
9.9689866 & & & 8.4085884 & 9.1313170 & 9.8587313 & \\
& & & 8.7855547 & 9.5750581 & & \\
& & & 9.3512139 & 9.9000521 & & \\
\hline
\end{tabular}

This table gives us the first zeros for the number fields of degree 5 and degree 6 of different signatures, having smallest discriminant. In each case, we give the signature and the discriminant of the field. We cannot prove the GRH for these fields because Theorem 4.4 assumes that we can find the zeros of $\zeta_{K}(s)$ up to a height 40 . 


\section{ACKNOWLEDGEMENTS}

I would like to thank Eduardo Friedman and Andrew Odlyzko for their interest in my work.

\section{REFERENCES}

1. M. Abramowitz and I.A. Stegun, Handbook of mathematical functions (with formulas, graphs, and mathematical tables), Dover Publications, Inc., New York, 1966. MR 34:8606

2. R. Backlund, Sur les zeros de la fonction $\zeta(s)$ de Riemann, C. R. Acad. Sci. Paris 158 (1914), 1979-1982.

3. B. L. J. Braaksma, Asymptotic expansions and analytic continuations for a class of Barnesintegrals, Compos. Math 15 (1964), 239-341. MR 29:4923

4. R. P. Brent, On the zeros of the Riemann zeta function in the critical strip, Math. Comp. 33 (1979), 1361-1372. MR 80g:10033

5. J. L. Duras, Etude de la fonction nombre de façons de représenter un entier comme produit de $k$ facteurs, Doctorat de l'Université de Limoges (1993).

6. H. M. Edwards, Riemann's zeta function, Academic Press, New York and London, 1974. MR $\mathbf{5 7 : 5 9 2 2}$

7. E. Friedman, Hecke's integral formula, Séminaire de Théorie des Nombres de Bordeaux 198788 (1988). MR 90i: 11136

8. E. Friedman, Analytic formulas for the regulator of a number field, Inventiones Math. 98 (1989), 599-622. MR 91c: 11061

9. E. Hecke, Über die Zetafunktion beliebiger algebraisher Zahlkörper, Gött. Nachr. (1917), 159171.

10. J. C. Lagarias and A. M. Odlyzko, On computing Artin L-functions in the critical strip, Math. Comp. 33 (1979), 1081-1095. MR 80g:12010

11. S. Lang, Algebraic number theory, Addison Wesley, 1970. MR 44:181

12. R. S. Lehman, On the distribution of the zeros of the Riemann zeta function, Proc. London Math. Soc. (3) 20 (1970), 303-320. MR 41:3414

13. R. S. Lehman, Separation of zeros of the Riemann zeta-function, Math. Comp. 20 (1966), 523-541. MR 34:3756

14. N. Nielsen, Der Theorie der Gamma-Funktion, Chelsea Publishing Company, 1965.

15. N. E. Nörlund, Vorlesungen über Differenzenrechnung, Springer, Berlin, 1924.

16. A. M. Odlyzko, Bounds for discriminants and related estimates for class numbers, regulators and zeros of zeta functions: a survey of recent results, Séminaire de Théorie des Nombres de Bordeaux 2 (1990), 119-141. MR 91i:11154

17. A. M. Odlyzko, Analytic computations in number theory, Proceedings of Symposia in Applied Mathematics (1994). MR 96a:11146

18. H. Rademacher, On the Phragmén-Lindelöf theorem and some applications, Math. Z. 72 (1959), 192-204. MR 22:7982

19. J. B. Rosser, L. Schonfeld and J. M. Yohe, Rigorous computations and the zeros of the Riemann zeta-function, Proceeding of IFIP Congress 68, 13-18.

20. R. Rumely, Numerical computations concerning the ERH, Math. Comp. 61 (1993), 415-440. MR 94b: 11085

21. A. M. Turing, Some calculations of the Riemann zeta function, Proc. London Math. Soc. (3) 3 (1953), 99-117. MR 14:1126e

U.M.R. 9936 Du C.N.R.S., U.F.R. De Mathématiques et Informatique, Université Bordeaux 1, 351 Cours de la libération, 33405 Talence Cedex, France

E-mail address: tollis@ecole.ceremab.u-bordeaux.fr 